\title{
LISTA COMENTADA DE ORCHIDACEAE EN URUGUAY Y SU DISTRIBUCIÓN EN AMBIENTES Y ECO-REGIONES
}

\author{
Lucía Marín Pérez ${ }^{1,5}$, Edlley M. Pessoa ${ }^{2,3} \&$ Marccus Alves ${ }^{4}$
}

${ }^{1}$ Programa de Posgrado en Biología Vegetal, Departamento de Botánica, Universidad Federal de Pernambuco, Profesor Moraes Rego, s.n. Ciudad Universitaria, 50.670-901, Recife, Pernambuco, Brasil

${ }^{2}$ Departamento de Botánica e Ecología, Instituto de Biociéncias, Universidad Federal de Mato Grosso, Cuiaba, MT, Brasil

${ }^{3}$ Programa de Posgrado en Biodiversidad, Ambiente y Salud, Universidad Estatal de Maranhão, Caxias, Maranhão, Brasil

${ }^{4}$ Departamento de Botánica, Universidad Federal de Pernambuco, Recife, Pernambuco, Brasil

${ }^{5}$ Author for correspondence: luciaperezbiologia@gmail.com

\begin{abstract}
Aвstract. From an economic and taxonomic point of view, Orchidaceae is one of the most studied botanical families. In Uruguay it is poorly represented and known, and descriptive data are fragmented and scattered. In the present work the information on the species of this family is updated based on herbaria records, previous publications and field trips made in the country during 2018-2019. A list of Orchidaceae from Uruguay is presented with 59 species belonging to 18 genera. Four species were found endemic to the country, while 22 species are endemic to the Southern Cone region. The Gondwanic Sedimentary Basin was the ecoregion with the highest species diversity (51\%) and the richest departments were Cerro Largo (25 spp.) and Maldonado (25 spp.). The Highland Forest and Riparian Forest environments presented highest diversity. The most representative genera were Habenaria (12 spp.), Cyclopogon (8 spp.), and Brachystele (6 spp.). The species with the widest distribution were Habenaria gourlieana, H. parviflora, and Cyclopogon elatus, which were found in all ecoregions and in 15 out of 19 departments of the country.
\end{abstract}

Resumen. Orchidaceae es una de las familias botánicas más estudiadas desde el punto de vista taxonómico y económico en el mundo. En Uruguay es poco conocida y los datos descriptivos que existen se encuentran fragmentados y dispersos. En este trabajo se presenta un inventario de la familia así como su distribución en ambientes, eco-regiones y departamentos del país basados en los registros de herbarios, publicaciones existentes y expediciones de campo realizadas durante el 2018-2019. Se presenta una lista con 59 especies en 18 géneros. Se encontraron cuatro especies endémicas de Uruguay, mientras que $22 \mathrm{spp}$. son endémicas del Cono Sur. La Cuenca Sedimentaria Gondwánica fue la eco-región en presentar mayor diversidad de especies (51\%) y los departamentos con mayor riqueza fueron Cerro Largo (25 spp.) y Maldonado ( $25 \mathrm{spp}$.). Mientras que el Bosques Serranos y Bosque Ribereño fueron los más ricos en especies de orquídeas. Los géneros más representativos fueron Habenaria (12 spp.), Cyclopogon (8 spp.) y Brachystele (6 spp.) y las especies que presentaron distribución amplia fueron Habenaria gourlieana, H. parviflora, y Cyclopogon elatus, las cuales se encontraron en todas las eco-regiones y en 15 de los 19 departamentos del país.

Key Words / Palabras clave: Cono Sur, Epidendroideae, Neotrópico, Neotropics, Orchidoideae, South America, Southern Cone, Sudamérica

Introducción. A nivel mundial, Orchidaceae (Asparagales, APG IV 2016) posee aproximadamente 28,000 especies, por lo que es considerada como una de las familias de plantas con mayor diversidad. Este grupo de plantas posee además, una amplia distribución, encontrándose en casi todas las regiones del planeta, con excepción de las zonas polares y desérticas (Dressler 1993, 2005, Christenhusz \& Byng 2016). Presenta características florales propias de la familia como lo es, la presencia de labelo, el polen aglutinado en sacos llamados polinios y la fusión de estambres y pistilo formando la columna 
(Cribb 1999, Dressler 2005). En la actualidad es uno de los grupos económicamente más importantes y en la industria de alimentos y cosméticos como saborizantes y aromatizantes (principalmente Vanilla planifolia Andrews) y entre las plantas ornamentales (Tan \& Chin 2015, Cameron 2011, Anilkumar 2004), y muchas de sus especies están categorizadas en algún nivel de amenaza (Swarts \& Dixon 2009).

En Uruguay son pocos los estudios existentes sobre la flora Orchidaceae, lo que conlleva a que sea una de las familias poco conocidas y, en los escasos registros que hay, se han encontrado algunas especies en ambientes con vegetación de pastizales, bosque de quebradas y serranías presentando colores poco vistosos en relación a la vegetación circundante y en comparación con las especies tropicales (Izaguirre 2010).

La flora del país fue revisada por Gibert (1873) y Arechavaleta (1894) indicando cinco géneros y ocho especies de Orchidaceae, seguidos por Herter (1930) con un catálogo florístico: Florula Uruguayensis mencionando 11 géneros y 21 especies. Con enfoque en Orchidaceae, Pabst (1952) menciona 45 especies en 12 géneros e Izaguirre (1985) 13 géneros con 47 especies y describe los cuatro géneros de hábito epífito conocidos (Campylocentrum Barb.Rodr., Capanemia Barb.Rodr., Oncidium Sw. y Pleurothallis R.Br.). Además, Izaguirre $(1972,1973)$ hace una revisión de los géneros Capanemia Barb.Rodr. y Bipinnula Comm. ex Juss. presentes en el país. En los últimos años, Izaguirre $(2010,2013)$ y Rossado et al. (2014) registran aproximadamente 60 especies en 18 géneros, siendo la mayoría de hábito terrestre y únicamente nueve epífitas. Entre ellas, 12 especies en nueve géneros son indicadas como nuevos registros para el país y Mai et al. (2019) publican un catalogo de la flora vascular epífita del país con 12 especies de la familia en siete géneros. En la región, el proyecto La Flora del Cono Sur (2020) menciona 132 géneros para el Cono Sur; mientras que Andrade et al. (2018) reportaron 149 especies en 54 géneros de Orchidaceae en los pastizales del Río de la Plata de la región Uruguayense (incluye Uruguay en su totalidad, el Sur de Río Grande del Sur de Brasil y el Este de la provincia de Entre Ríos en Argentina) de los cuales 19 géneros con 57 especies pertenecen a Uruguay.

En la Lista de Especies Prioritarias para la Conservación del País, publicado por Marchesi et al. (2013), citan 29 especies de orquídeas con algún riesgo de extinción basados en criterios de raridad (especies colectadas pocas veces), con una significativa disminución en la población ya sea por actividades antropogénicas, distribución restringida y/o endemismo en el país.

Constantemente en Uruguay hay un aumento en la expansión de suelos para la agricultura, ganadería, forestación y/o expansión urbana (Achkar et al. 2012), lo que afecta considerablemente la diversidad de orquídeas en la región, aunado a esto que los estudios en Orchidaceae son muy escasos conlleva a que aproximadamente el $50 \%$ del total de las especies registradas están bajo algún grado de amenaza (Marchesi et al. 2013). Es por ello que Orchidaceae requiere de estudios florísticos, taxonómicos y geográficos que permitan crear una estrategia a nivel nacional que resulte eficaz para contribuir en su conocimiento, la conservación de su diversidad y hábitat. En este sentido el objetivo de este trabajo fue recopilar la información de las orquídeas de Uruguay para elaborar un documento donde se disponga de un listado actualizado de las especies presentes y que permitan estudiar aspectos de su distribución en el país en relación a los ambientes y eco-regiones que ocupan.

\section{Materiales y métodos}

Fitogeografia de Uruguay.- Uruguay esta ubicado en el Cono Sur sudamericano, limitando al Oeste con Argentina, y al Norte con Brasil. Su superficie continental es de $176.215 \mathrm{~km}^{2}$, con costas al Sur sobre el Río de la Plata (452 km de longitud) y al Este sobre el Océano Atlántico $(228 \mathrm{~km})$ (Instituto Geográfico Militar [IGM] 2020). La red hidrográfica es extensa, con seis cuencas hidrográficas principales: Río Negro, Río de la Plata, Río Santa Lucía, Río Uruguay (asociadas al Río de la Plata) y Laguna Merín, y Océano Atlántico (asociadas al Océano Atlántico). Se destaca el Río Santa Lucía por ser la principal abastecedora de agua potable para la capital y zona metropolitana (aproximadamente el $50 \%$ de la población total) (MVOTMA-Geoservicios 2020b). Se encuentra en una zona climática templada, con precipitación media anual de $1300 \mathrm{~mm}$ y temperatura media de $17.5^{\circ} \mathrm{C}$ (IGM 2020, Instituto Uruguayo de Meteorología [INUMET] 2019). Presenta una división administrativa organizada en 19 departamentos: Artigas (ART), Canelones 


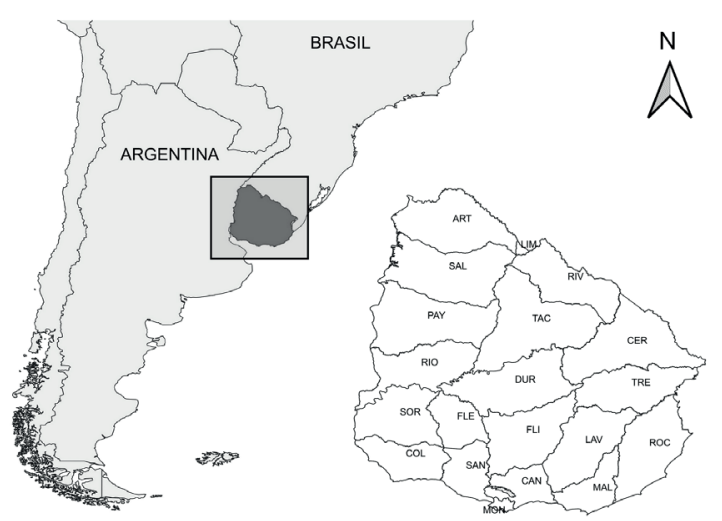

FIgura 1. División administrativa de Uruguay. Departamentos: Artigas (ART), Canelones (CAN), Cerro Largo (CER), Colonia (COL), Durazno (DUR), Flores (FLE), Florida (FLI), Lavalleja (LAV), Limite Contestado (LIM), Maldonado (MAL), Montevideo (MON), Paysandu (PAY), Rio Negro (RIO), Rivera (RIV), Rocha (ROC), Salto (SAL), San Jose (SAN), Soriano (SOR), Tacuarembo (TAC) y Treinta y Tres (TRE). Mapa elaborado por L. Marín [departamentos: MVOTMA-Geoservicios. (2020a)].

(CAN), Cerro Largo (CER), Colonia (COL), Durazno (DUR), Flores (FLE), Florida (FLI), Lavalleja (LAV), Maldonado (MAL), Montevideo (MON), Paysandú (PAY), Río Negro (RIO), Rivera (RIV), Rocha (ROC), Salto (SAL), San José (SAN), Soriano (SOR), Tacuarembó (TAC) y Treinta y Tres (TRE) (Fig. 1).

Los ambientes del país (Fig. 2) son considerados como zonas de transición con una alta influencia de la vegetación de las provincias vecinas Paranaense y Chaqueña; presenta además, una considerable biodiversidad (Cabrera \& Willink 1973, Morrone 2001, Grela 2004). En éstos departamentos, las principales actividades económicas realizadas es la producción ganadera y la agrícola, las cuales se ven favorecidas por la predominancia de ambientes de pastizales (MGAP 2015). Las actividades derivadas de estas prácticas (defoliación, pisoteo, selectividad y redistribución de nutrientes) han demostrado una importante perturbación en la dinámica, en la composición de pastizales y de los bosques, resultando negativa cuando se realiza con una alta intensidad (Orden et al. 2006, Zhao et al. 2007, Tálamo et al. 2009, Chemisquy 2013). Sin embargo, diversos estudios (Gonçalves 2016, García et al. 2019) indican que algunos componentes bajo determinadas intensidades cumplen un papel importante en la subcistencia y el aumento de la biodiversidad.

Los pastizales (P), están formados por vegetación campestre y ocupan aproximadamente $88 \%$ del territorio nacional (Blum 2015) esto hace que el país sea considerado dentro de la eco-región de Pastizales del Río de la Plata (región Uruguayense) (Andrade et al. 2018). Además, forma parte de una de las ecoregiones continuas más grande de América (Soriano 1992, Dixon et al. 2014).

La vegetación boscosa representa aproximadamente $5.2 \%$ del territorio nacional (MGAP 2018) y está formada por Bosques (B) clasificados en función de la ubicación y características de la vegetación (Chebataroff 1960, Del Puerto 1987, Brussa 1996, Evia \& Gudynas 2000, Rivas 2010, Panario et al. 2015) en: 1. Bosques Ribereños o Fluviales (BR), presentan franjas de vegetación arbórea que acompañan los cursos de agua de todo el país; 2. Bosques de Quebrada (BQ), se desarrollan en cañones profundos asociados a los cursos de agua que existen en el norte del país; 3. Bosques Serranos (BS), ubicados en las laderas de las sierras, con vegetación arbustiva; 4. Bosques de Parque (BP), se encuentran en el litoral oeste y centro sur, presenta una densidad relativamente baja de árboles y representa un tipo de comunidad sub-xerófila compuesta por espinillos (Vachellia caven (Molina) Seigler \& Ebinger - Fabaceae) y algarrobos (Prosopis nigra Hieron. - Fabaceae); 5. Palmares (BPa), presentan distribución restringida encontrándose únicamente dentro del país, donde predomina la vegetación arborea como palmeras, Butia capitata (Mart.) Becc. (Arecaceae) en el departamento de ROC y Butia yatay (Mart.) Becc. (Arecaceae) en PAY y RIO; 6. Bosques Costeros (BC), con vegetación psamófila, ubicados en las costas del Río de la Plata y Océano Atlántico, éstos últimos asociados a sistemas de lagunas costeras (José Ignacio, Garzón, Rocha y Castillos).

En estos ambientes existen zonas bajas húmedas denominadas Humedales o Bañados $(\mathrm{H})$, caracterizados como tierras bajas inundadas ya sea de forma esporádica o permanente, en ellos la vegetación está formada por plantas hidrófilas (Evia \& Gudynas 2000, Rivas 2010, Panario et al. 2015).

El territorio uruguayo presenta siete eco-regiones basadas en variables geoambientales (relieve, edáficas) y biológicas (riqueza de especies, ambientes dominantes) (Brazeiro et al.2015a). Estas eco-regiones 

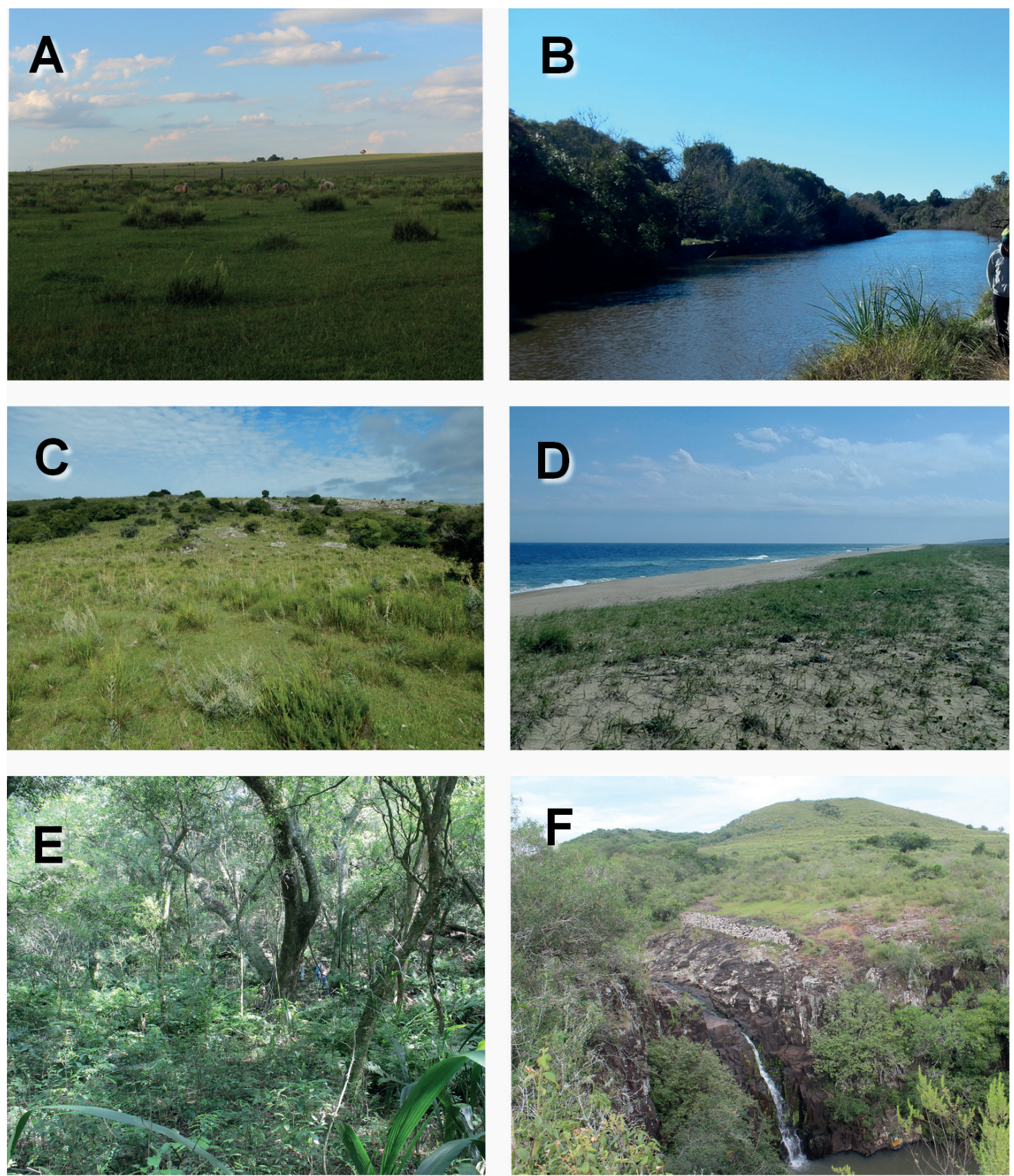

Figura 2. Ambientes de Uruguay. A: Pastizales (P). B: Bosques Ribereños (BR). C: Bosques Serranos (BS). D: Bosques

Costeros (BC). E, F: Bosques de Quebrada (BQ). Fotografías por L. Marín.

(Tabla 1) según Brazeiro et al. (2015a) son: 1. Cuesta Basáltica $(\mathrm{CuBa})$, se ubica al norte del país, presentando mayor extensión y mayor riqueza de especies; en la cual predominan los ambientes $\mathrm{P}, \mathrm{BR}$ y BQ. Además se encuentran relieves de hasta $400 \mathrm{~m}$ s.n.m.; 2. Cuenca
Sedimentaria Gondwánica (CuSeGo), se localiza al noreste del país, en ella predominan ambientes BQ y relieves con elevaciones de 380 m s.n.m.; además tienen prioridad de conservación por ser una de las superficies con mayor área (14\% de la eco-región); 3. Cuenca 
TABLA 1. Departamentos, ambientes y relieve según Brazeiro et al. (2015) y siglas de cada Eco-región del país.

\begin{tabular}{l|c|l|l|c}
\hline Eco-región & Sigla & Departamentos & Ambientes & Relieve (m sm) \\
\hline $\begin{array}{l}\text { Eco-región } \\
\text { Cuesta Basáltica }\end{array}$ & CuBa & ART, SAL, PAY, RIO, TAC, DUR, RIV & P, BR, BQ, BS & $20-400$ \\
\hline $\begin{array}{l}\text { Cuenca Sedimentaria } \\
\text { Gondwánica }\end{array}$ & CuSeGo & RIV, TAC, LAV, DUR & BQ, BS & $90-380$ \\
\hline $\begin{array}{l}\text { Cuenca Sedimentaria del } \\
\text { Oeste }\end{array}$ & CuSeOe & ART, SAL, PAY, RIO, SOR, COL & BR, BP & $5-160$ \\
\hline Escudo Cristalino & EsCr & COL, SOR, FLE, FLI, SAN, DUR & P, BP, BR & $0-190$ \\
\hline Graben del Santa Lucía & GrSaLu & MON, CAN, LAV, FLI, SAN & BC, P, H, BP & $0-120$ \\
\hline Sierras del Este & SiEs & $\begin{array}{l}\text { CAN, MAL, LAV, FLI, ROC, TRE, } \\
\text { CER, DUR }\end{array}$ & BS & $0-500$ \\
\hline Graben de la Laguna Merín & GrLaMe & MAL, ROC, LAV, TRE, CER & H, BPa, BC & $0-40$ \\
\hline
\end{tabular}

Sedimentaria del Oeste (CuSeOe), se ubica en el litoral oeste del país donde los principales ambientes son BR y BP; 4. Escudo Cristalino (EsCr), situada en el suroeste, cuyos ambientes principales son P, BP y BC.; ademas, presenta superficies de alta prioridad de conservación (19\% de la eco-región); 5. Graben de Santa Lucía (GrSaLu), se localiza al sur del país,es la región con mayor grado de urbanización y población. Esta zona en comparación con las otras eco-regiones se caracteriza por presentar menor área de extensión con una escasa riqueza de especies. Dentro de esta región se pueden encontrar ambientes como P, BP, BC y H. Además, es considerada como una superficie con alta prioridad de conservación (15\% de la eco-región); 6. Sierras del Este (SiEs), ubicada al este del país, específicamente en la región de Sierras, presenta relieves $500 \mathrm{~m}$ s.m. y esta constituida principalmente por ambientes de BS. Además, en esta eco-región se dan importantes nacientes de ríos del país; 7. Graben de la Laguna Merín (GrLaMe), situada en la costa este del país, los ambientes principales son $\mathrm{H}, \mathrm{BPa}$ y $\mathrm{BC}$, poseeuna de las regiones con mayor superficie con alta prioridad de conservación (19\% de la eco-región).

Todas las eco-regiones del país están siendo amenazas principalmente por actividades como la agricultura (soja y trigo) y la ganadería (principales actividades productivas) los cuales ocupan el $90 \%$ del territorio, afectando todos los ambientes de pastizales, bosques y los humedales (MGAP 2015). Otros de los factores que está contribuyendo negativamente al deterioro de estos ambientes son la deforestación (industria maderera), extracción minera, invasiones biológicas en bosques,en pastizales y la expansión urbana (Brazeiro, Soutullo \& Bartesaghi 2015b).

Colecta de Datos.- El presente inventario de especies se obtuvo mediante una amplia revisión de las publicaciones sobre Orchidaceae de la región Uruguayense (Herter 1930, Pabst 1952, Correa 1953, 1955, 1969, Izaguirre 1972, 1973, 1985, 2010, 2013, Pabst \& Dungs 1977, 1975, Garay 1982, Lombardo 1984, Szlachetko 1996, Pridgeon et al. 1999, 2003, Szlachetko, Gonzalez-Tamayo \& Rutkowski 2001, Hurrel et al. 2009, Salazar et al. 2009, 2018, MytnikEjsmont, Szlachetko \& Gorniak 2010, Schinini 2010, Batista, Bianchetti, Gonzalez-Tamayo, Figueroa \& Cribb 2011a, 2011b, Buzatto, Singer, Romero-Gonzalez, Van den Berg \& Salazar 2013, 2014, Marchesi et al. 2013, De Azevedo,Van Den Berg \& De Barros, 2014, Martos, Johnson, Peter \& Bytebier 2014, Radins, Salazar, Cabrera, Jimenez-Machorro \& Batista 2014, Rossado, Mai, Bonifacino \& Waechter 2014, Andrade et al. 2018, Castro \& Singer 2018, Mai et al. 2019, Pessoa \& Alves 2019, Buzatto, Singer, van den Berg, de Souza \& Mota 2020), bases de datos virtuales como la Flora del Cono Sur (2020), Govaerts et al. (2020), IPNI (2020), Reflora (2020), The Plant List (2020), Tropicos (2020) y visitas a los herbarios del país y extranjeros de relevancia para el grupo, BAF, $\mathrm{HBG}^{*}, \mathrm{ICN}^{*}, \mathrm{~K}^{*}$, MVFA, MVFQ, MVHC, MVJB, MVM, P*, SI, SP* (“*” consulta virtual). Se consultaron bases de datos en línea y literatura para revisión de la nomenclatura y obtener la distribución de las especies en países de América del Sur. 

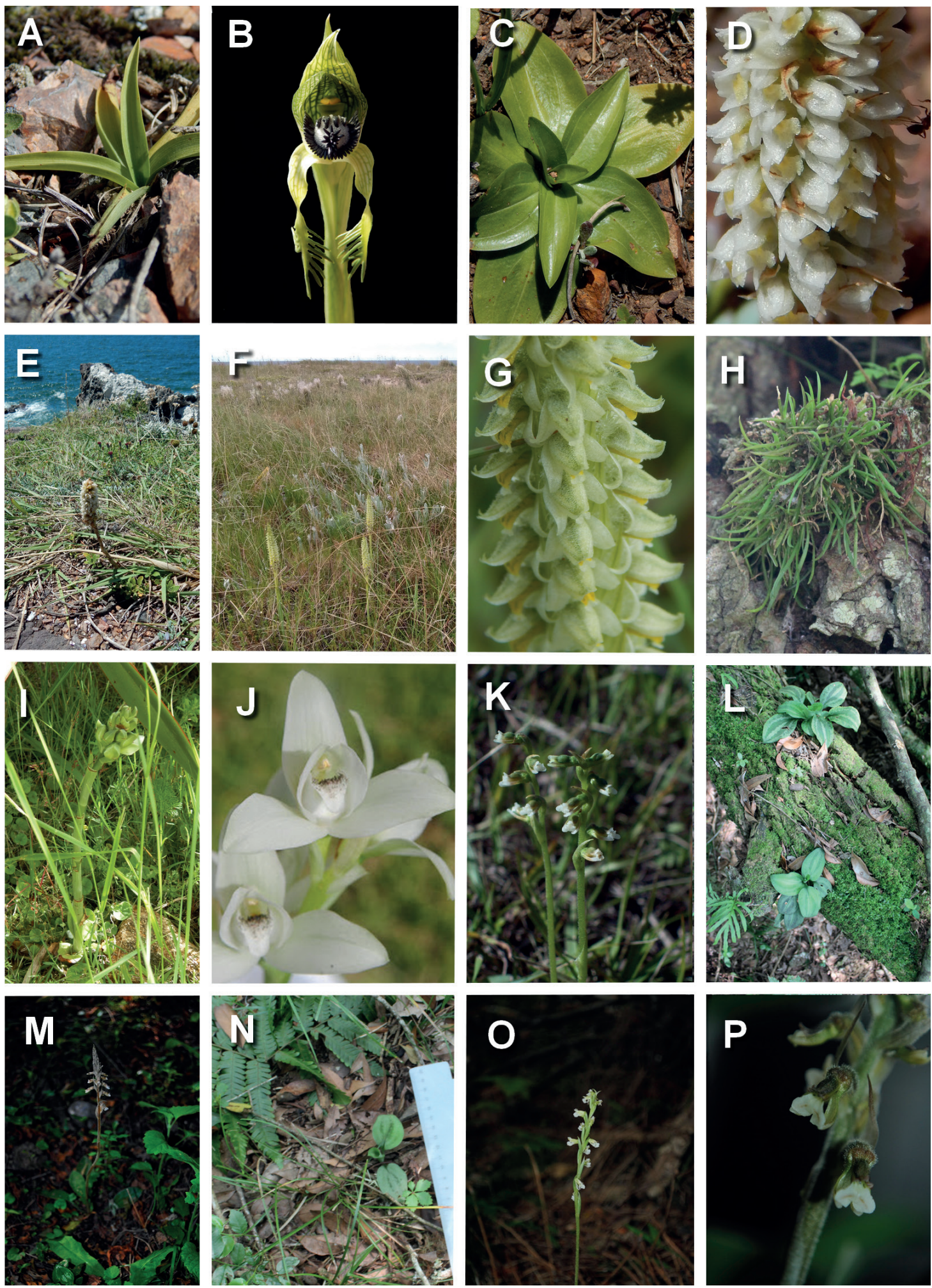
TABLA 2. Especies endémicas del Uruguay y departamentos, ambientes y eco-regiones en los que ocurren [Lavalleja (LAV), Maldonado (MAL), Montevideo (MON), Rocha (ROC); Pastizales (P), Bosques Ribereños (BR), Bosques Serranos (BS), Bosques Costeros (BC); Graben de Santa Lucía (GrSaLu), Sierras del Este (SiEs), Graben de la Laguna Merín $(\mathrm{GrLaMe})]$.

\begin{tabular}{l|l|l|l}
\hline Especie & Eco-regiones & Departamentos & Ambientes \\
\hline Brachystele pappulosa & GrSaLu & MON & - \\
\hline Brachystele waldemarii & SiEs & LAV, MAL & BS \\
\hline Pelexia arechavaletae & GrSaLu & MON & BR \\
\hline Skeptrostachys berroana & SiEs, GrLaMe & LAV, MAL, ROC & BC, BS, P \\
\hline
\end{tabular}

Para ampliar la información recopilada se realizaron 30 colectas de campo en Uruguay entre Noviembre de 2018 y Marzo 2019 en localidades donde existían registros previos de acuerdo con la revisión bibliográfica e información registrada en Vouchers. Estas abarcaron zonas dentro de las ecoregiones $\mathrm{CuSeGo}, \mathrm{CuSeOe}, \mathrm{EsCr}, \mathrm{GrSaLu}, \mathrm{SiEs}$ y GrLaMe y diversos ambientes (BR, BC, BS, BQ) en los departamentos MON, CAN, MAL, ROC, TAC, SOR y COL. Las muestras colectadas fueron procesadas y depositadas en MVJB.

Se elaboró un listado donde se indica el basiónimo y sinónimos más usados, hábito, endemismo en Uruguay y estatus de prioridad de conservación siguiendo la Lista de Especies Prioritarias para la Conservación del país de Marchesi et al. (2013). Además se incluyen notas indicando cambios nomenclaturales y principales obras donde se menciona la distribución de las especies en el país. En caso de encontrarse la misma especie repetidas en una misma zona, se colocó un solo ejemplar por cada departamento de ocurrencia del país. Los taxones que no fueron publicados como ocurrencia para el país o especímenes depositados en los herbarios de los cuales no fue posible verificar la procedencia de coleta no fueron incluidos.

La distribución en Uruguay es presentada por departamentos, ambientes y eco-regiones de ocurrencia. Los mapas fueron elaborados en el software QGIS-GRASS 7.6.1 con bases de datos físicos del país de Geoservicios - MVOTMA (2020), eco-regiones de Brazeiro et al. (2015a), ambientes de Evia \& Gudynas (2000) y la ocurrencia de especies de acuerdo a los registros de especies depositados en herbarios, literatura, bases de datos en línea y colectas realizadas.

Resultados y Discusión. Se obtuvo que la familia Orchidaceae en Uruguay está representada por 59 especies en 18 géneros (Fig. 3-4). Entre ellos, 48 especies son de hábito terrestre, 10 presentan hábito epifito y una especie rupícola (Cyrtopodium brandonianum Barb.Rodr.) (Fig. 5 A-B). Además, se obtuvo un total de cuatro especies endémicas, distribuidas en los ambientes BR, BS, BC y P (Tabla 2), $37 \%$ (22 spp.) se encontraron restringidas en la zona del Cono Sur (Argentina, sur de Brasil y Uruguay) y 61\% (36 spp.) considerando a Paraguay [utilizando el concepto de endemismo con flexibilización de los criterio de restricción, dentro de los límites administrativos de un país (De Candolle 1855, Ferreira \& Boldrini 2011)]. De las 5 subfamilias que existen a nivel mundial, se obtuvo representantes de dos subfamilias Epidendroideae con 15 especies $(25 \%)$ y Orchidoideae con 44 especies (75\%) (Fig. 6). Donde Orchidoideae resultó ser la más representativa ya que presentó especies de tres tribus que son: Chloraeeae (7 spp.), Orchideae (12 spp.) y Cranichideae (25 spp.). Dentro de Cranichideae la mayor subtribu es Spiranthinae con 23 especies (39\%).

Los géneros que presentaron mayor cantidad de especies fueron Habenaria Willd. (12 spp.), Cyclopogon C.Presl. (8 spp.), Brachystele Schltr. (6 spp.), Bipinnula Comm. ex Juss. (5 spp.) y Skeptrostachys Garay (5 spp.). Además se obtuvo que

A la izquierda, Figura 3. Orchidaceae de Uruguay. A: Bipinnula montana, hojas. B: B. montana. C: Brachystele camporum, hojas. D: B. camporum. E: B. camporum, hábito. F: Brachystele dilatata, hábito. G: B. dilatata. H: Capanemia micromera. I: Chloraea membranaceae, hábito. J: C. membranaceae. K: Cyclopogon apricus. L: Cyclopogon sp., hábito epífito. M: Cyclopogon elatus, inflorescencia. N: C. elatus, hojas. O: C. micrantha. P: C. chloroleucus. Fotografías por L. Marín (A-I, L, N) y A. González (J, K, M, O, P). 

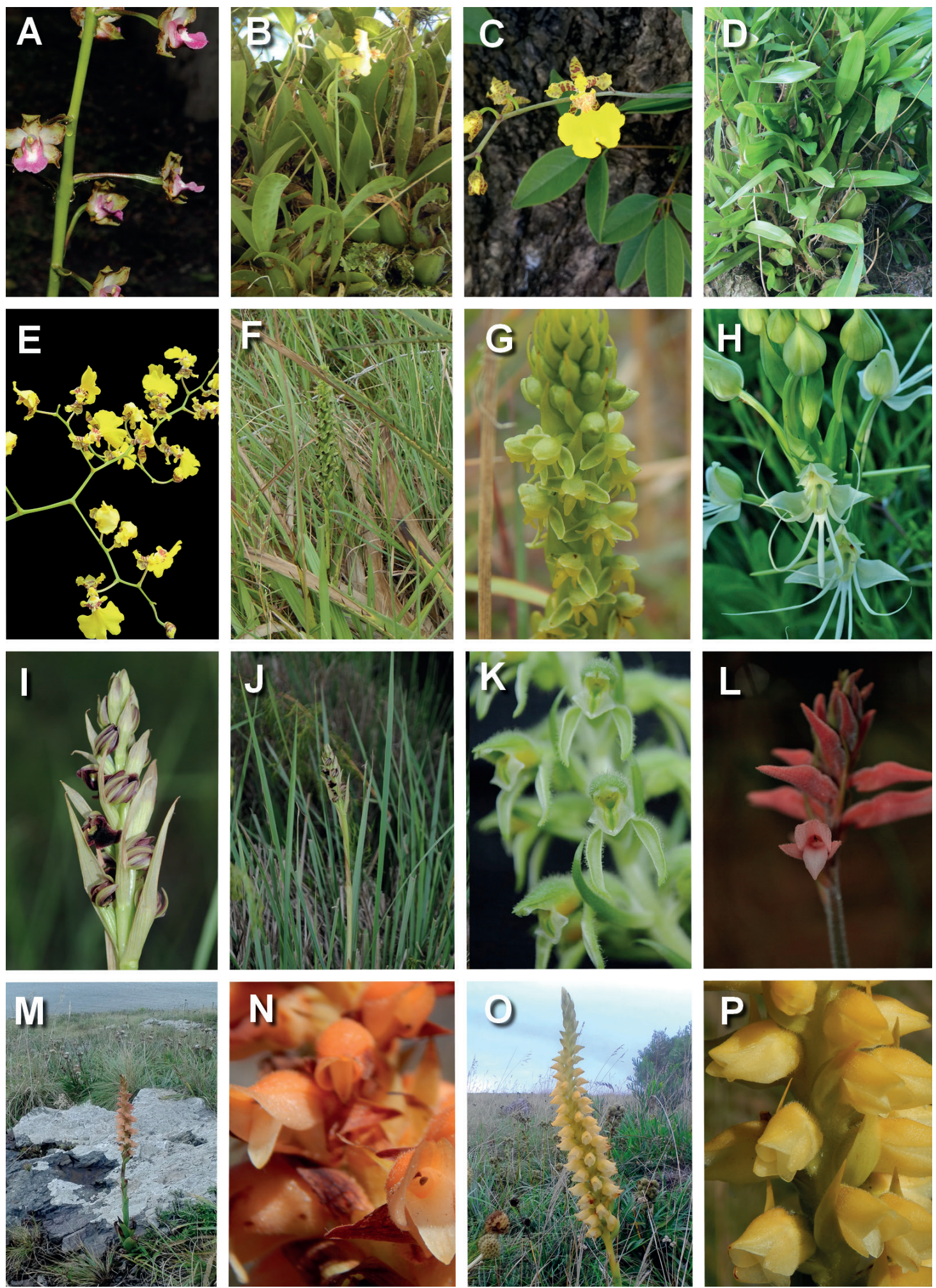
A

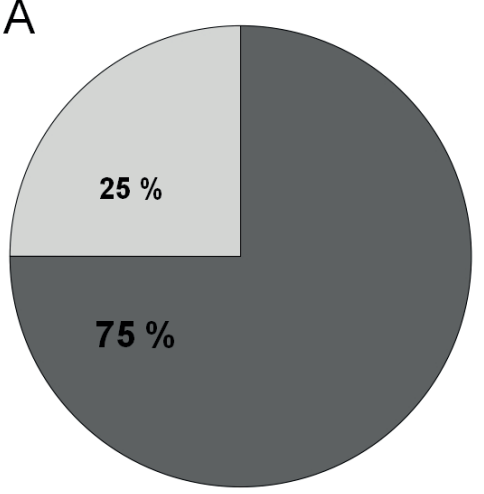

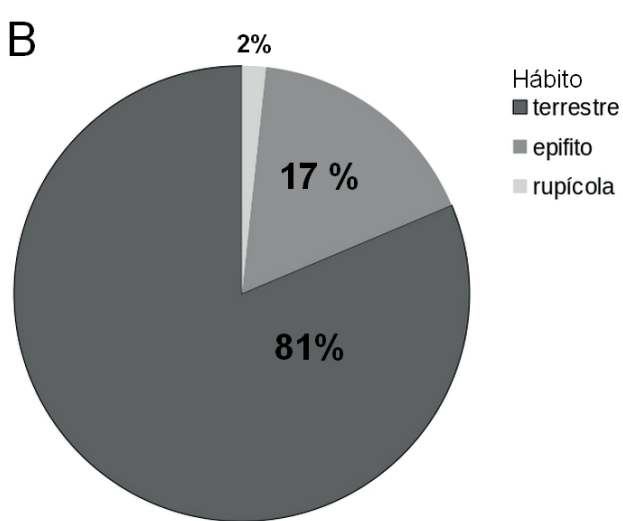

Figura 5. A: Riqueza de especies de las subfamilias Orchidoideae y Epidendroideae registradas en Uruguay. B: Hábitos de crecimiento de las orquídeas de Uruguay.

un 33\% de los géneros presentes en el país solo están representados por una especie; mientras que el $72 \%$ restantes incluyen hasta un máximo de tres especies (Fig. 7 A-B).

Las especies Habenaria parviflora Lindl. (16 departamentos), Habenaria gourlieana Gillies ex Lindl. (15 departamentos) y Cyclopogon elatus (Sw.) Schltr. (15 departamentos), presentaron mayor distribución ya que se encontraron en todas las ecoregiones del país.

Los ambientes que reflejaron mayor diversidad (Fig. 8 y 9, Anexo 1) fueron BS (61\%) y BR (53\%) seguidos de BQ (32\%), BC (31\%). La posibilidad de sobrevivencia de especies de hábito terrestre y epifito son factores que pueden estar relacionados, siendo encontradas en estos ambientes 5 de las 10 especies epifitas en BS y 9 spp. en BR. La existencia de legislaciones antiguas de protección a bosques nativos (Ley $\mathrm{N}^{\circ} 15939,1987$ ) colaboran de forma parcial en su conservación (Rivas 2010). Por otro lado, la mayoría (20 spp.) de las especies que ocurren son de amplia distribución o pueden encontrarse en otros ambientes como BQ y BR. La eco-región que presentó menor diversidad y riqueza de especies fue $\mathrm{BPa}(3 \%)$ siendo también uno de los ambientes de menor extensión en el país (Evia \& Gudynas 2000) que junto con las zonas húmedas, es intensamente amenazado por las prácticas de agicultura como el cultivo de arroz y el sobrepastoreo en la ganadería (Rivas 2010).

En relación a los departamentos, los que presentaron mayor diversidad de especies fueron, CER (25 spp. en 11 géneros) y MAL ( 25 spp. en 8 géneros), mientras que ART (4 spp. en 3 géneros) y FLE (1 spp.) presentaron menor diversidad (Fig. 10, Anexo 2).

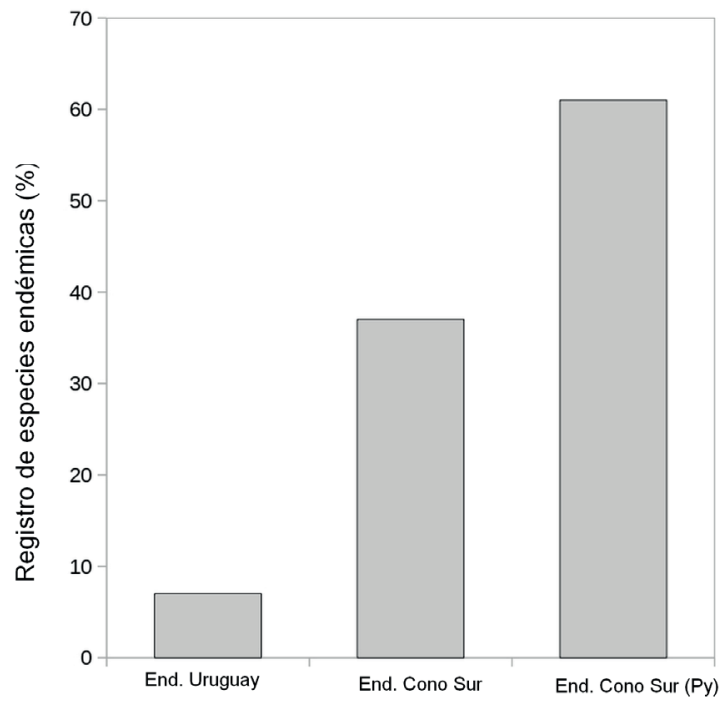

Figura 6. Riqueza de orquídeas endémicas de Uruguay, restringidas al Cono Sur (Argentina, sur de Brasil y Uruguay) y considerando a Paraguay.

A la izquierda, Figura 4. Orchidaceae de Uruguay. A: Cyrtopodium brandonianum. B: Gomesa bifolia, hábito. C: G. bifolia. D: G. flexuosa, hábito. E: G. flexuosa. F: Habenaria parviflora, hábito. G: H. parviflora. H: H. gourlieana. I: Orthochilus ruwenzoriensis. J: O. ruwenzoriensis, hábito. K: Pelexia bonariensis. L: Sacolia lanceolata. M: Skeptrostachys arechavaletanii, hábito. N: S. arechavaletanii. O: S. gigantea, hábito. P: S. gigantea. Fotografías por A. González (A, H-L) y L. Marín (B-G, M-P). 


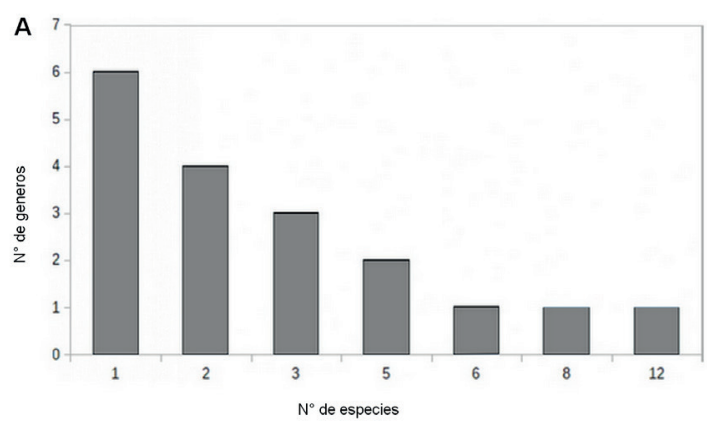

B

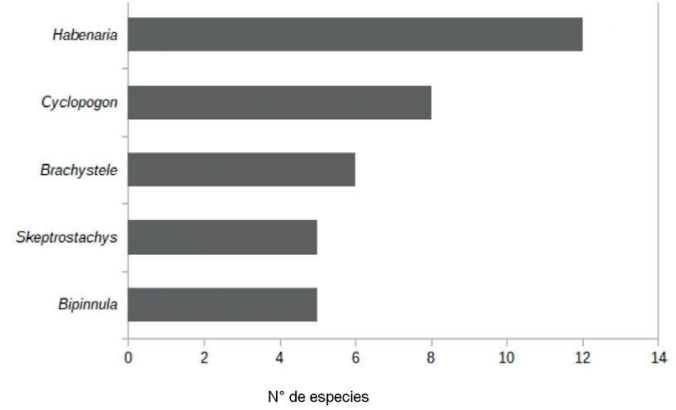

Figura 7. A: Diversidad de especies en los géneros de Uruguay. B: Géneros de orquídeas más diversos en el país.

Las eco-regiones que presentaron mayor riqueza de especies fueron CuSeGo con 51\% (30 spp. en 11 géneros), GrLaMe con $42 \%$ ( 25 spp. en 10 géneros) y GrSaLu con $42 \%$ (25 spp. en 8 géneros), mientras que EsCr (14 spp. en 6 géneros) y CuSeOe (13 spp. en 7 géneros) reflejaron menor riqueza de especies (Fig.11, Anexo 3).

Esta diferencia en CuSeGo, principalmente en los departamentos CER, RIV y TAC, y en GrLaMe en MAL, ROC y LAV coincide con la reportado en otros grupos biológicos (Grela 2004, Perez-Quesada \& Brazeiro 2013, Mai 2014). Además, CuSeGo es la segunda con mayor riqueza de especies leñosas y epifitas del país (Mai 2014, Brazeiro et al. 2015a) también concuerda con el núcleo norte de flora leñosa (Grela 2004). En GrLaMe, cabe resaltar que la mayoría de las especies encontradas principalmente en MAL son de amplia distribución.

El aumento de las riquezas en las eco-regiones CuSeGo y GrLaMe puede verse posiblemente favorecida por los factores como: la dispersión de especies provenientes de la flora de las Provincias vecinas como Paranaense, Chaqueña y/o Cerrado, a través de las Quebradas del Norte y Sierras del Este, ocasionando un aumento en el límite de distribución de diversos grupos hacia el sur (Grela \& Brussa 2007). Esto, a su vez también esta asociado al relieve, con la formación de microclimas, elevadas temperaturas y bancos de niebla los cuales mantienen constante la humedad en la zona de CuSeGo y una alta pluviosidad promedio en GrLaMe permitiendo que haya variabilidad dentro de los ambientes (Grela \& Brussa 2007, Mai 2014) (Fig. 12).

Por otro lado, CuSeOe (22\%) y EsCr (24\%), son las eco-regiones con menor número de especies y es donde se encuentra la mayoría de departamentos con menor cantidad de especies DUR (5 spp. en 4 géneros), PAY (5 spp. en 4 géneros) y FLE (1 sp.). Esta menor riqueza puede estar relacionada a la predominancia de Py BP en los que fueron encontrados el $29 \%$ y $22 \%$ de especies. Además de la degradación ambiental de la zona. La superficie en $\mathrm{CuSeOe}$ considerada de alta prioridad de conservación es de 19\%, mientras que en EsCr es de 10\% (Brazeiro et al. 2015b) y la presión por el uso del suelo en agricultura intensiva (trigo-soja) que llega a $26 \%$ en EsCr y $19 \%$ en CuSeOe (Achkar et al. 2015). Esta actividad es una de las principales prácticas que causan un fuerte impacto en las poblaciones de especies endémicas de la región, ya que, en los cultivos se utilizan diferentes compuestos químicos como herbicidas, fungicidas y/o fertilizantes (Schrag et al. 2009, Achkar et al. 2015). En cambio en las zonas como CuBa (19 spp.) donde predominan actividades de pastoreo, se ha encontrado que son menos perjudiciales (en comparación con la agricultura intensiva) para la subsistencia de la diversidad (Dostálek \& Frantik 2008, García et al. 2019) incluso en Orchidaceae (Gonçalves 2016).

Las eco-regiones como GrLaMe y ambientes similares a BQ de CuSeGo presentan mayores registros de herbarios, esto podría estar relacionadocon la accesibilidad de sus sitios de colectas, los cuales se encontraron en zonas pobladas o lugares particulares con heterogeneidad ambiental como algunas áreas de conservación con diversos ambientes juntos como BR, BS y BQ en el Arroyo Lunarejo, Arroyo Laureles, Paso Centurión o Sierra de Ríos (Haretche et al. 2012, Mai 2013). Esto pudo favorecer la alta riqueza de especies registrada. Además, se encontró mayor cantidad de registros en $\mathrm{BC}$ relacionados con la zona turística de la costa atlántica, el Río de la Plata y con 


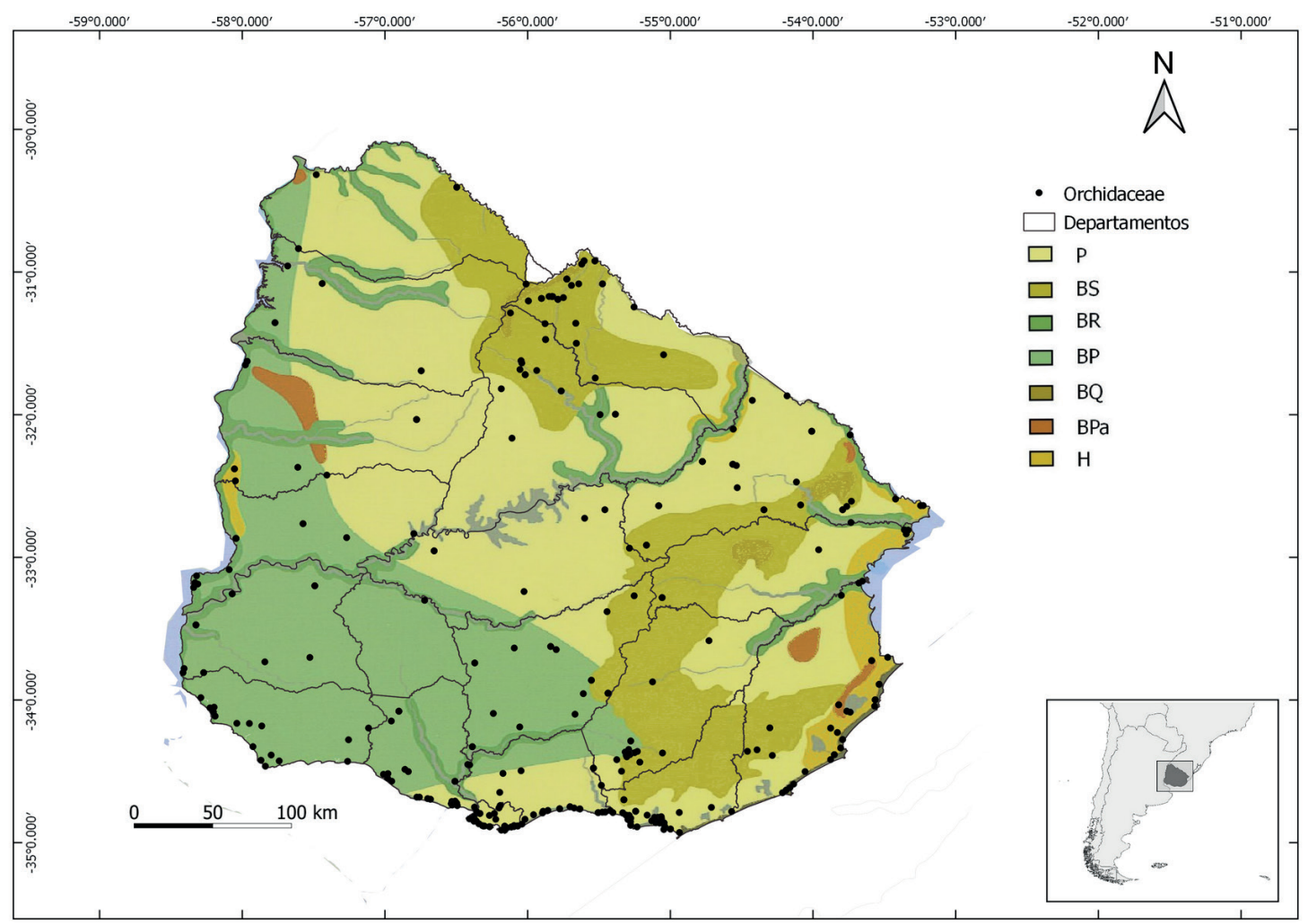

Figura 8. Distribución de Orchidaceae en Ambientes del Uruguay [Pastizales (P), Bosques Ribereños (BR), Bosques de Quebrada (BQ), Bosques Serranos (BS), Bosques de Parque (BP), Bosques Costeros (BC), Palmares (BPa) y Humedales (H)]. Mapa elaborado por L. Marín [departamentos: MVOTMA-Geoservicios. (2020a); ambientes adaptado de Evia \& Gudynas (2000)].

la zona metropolitana ( $\mathrm{GrSaLu}$ asociado a la capital del país) que presentan mayor facilidad de acceso a los ambientes.

En áreas de conservación, con base en los análisis de los mapas de ocurrencia de especies en relación al Sistema Nacional de Áreas Protegidas (SNAP 2020), fueron encontradas 29 especies, sobresaliendo los Humedales de Santa Lucía (13 spp.), Valle del Lunarejo y Laureles Cañas (11 spp.) y Montes del Queguay (9 spp.). En total ca. del 50\% de las especies esta representada en alguna de estas área, resaltando así, la importancia de la conservación de Orchidaceae (Fig. 13).

En relación a plantas vasculares, Orchidaceae es una de las familias que presenta mayor cantidad de especies prioritarias para conservación (29 spp.) del país y la única con una especie (Bipinnula biplumata) con los cuatro criterios de vulnerabilidad de Marchesi et al. (2013). Estas especies se encontraron principalmente en CuSeGo 39\%, GrLaMe 39\% y $\mathrm{CuBa} 36 \%$ siguiendo las tendencias en otros grupos biológicos donde existe una correlación positiva entre la riqueza total de especies y las que presentan amenaza

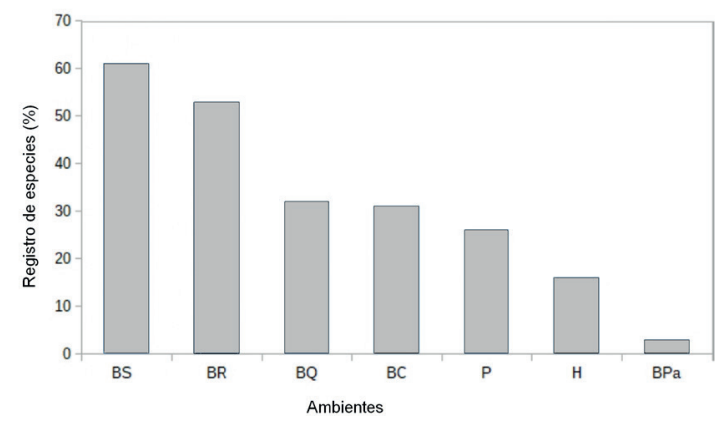

Figura 9. Riqueza de especies registradas en ambientes de Uruguay [Pastizales (P), Bosques Ribereños (BR), Bosques de Quebrada (BQ), Bosques Serranos (BS), Bosques de Parque (BP), Bosques Costeros (BC), Palmares $(\mathrm{BPa})$ y Humedales $(\mathrm{H})]$. 


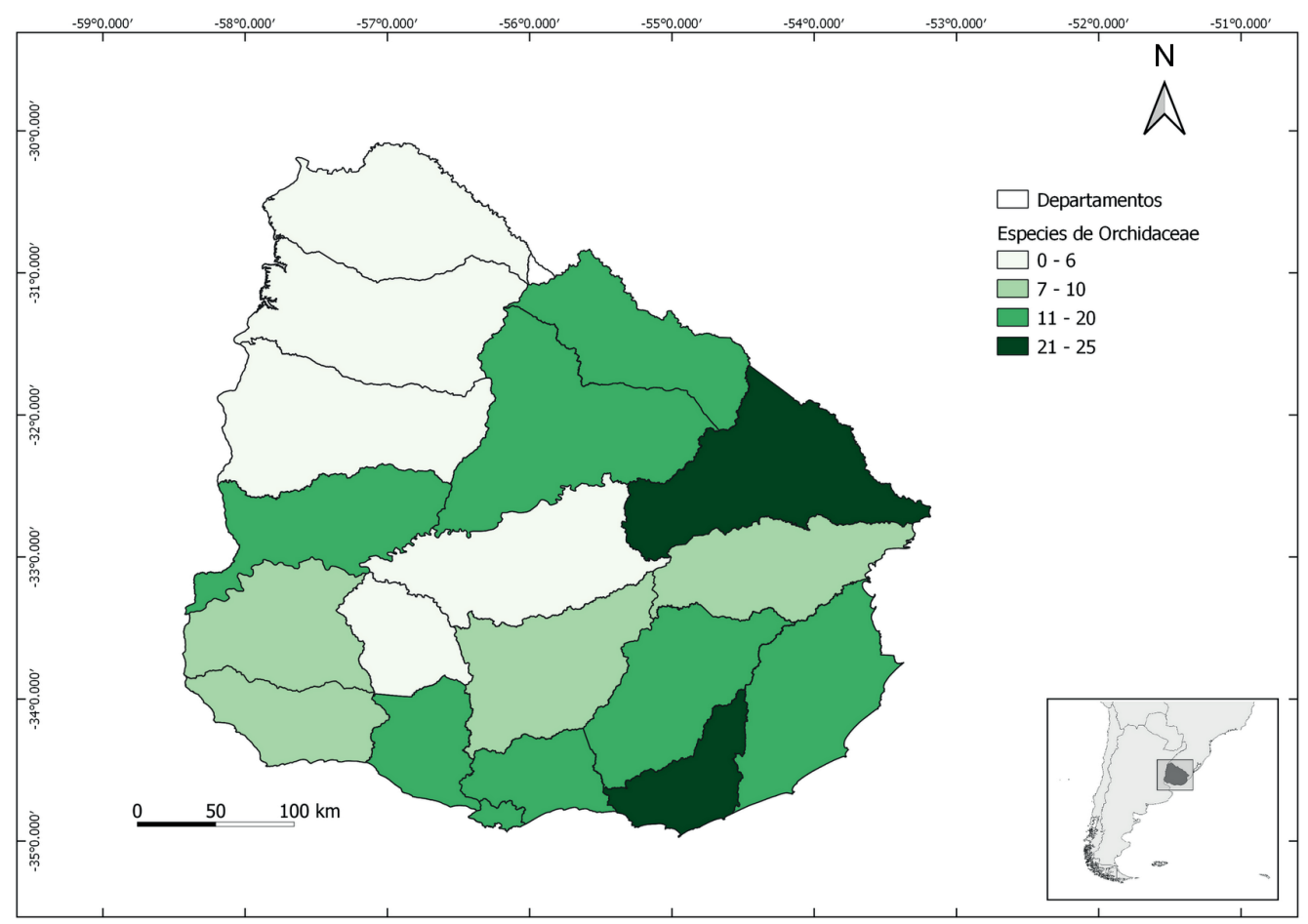

FiguRA 10. Riqueza de Orchidaceae en los departamentos de Uruguay. Mapa elaborado por L. Marín [departamentos: MVOTMA-Geoservicios. (2020a)].

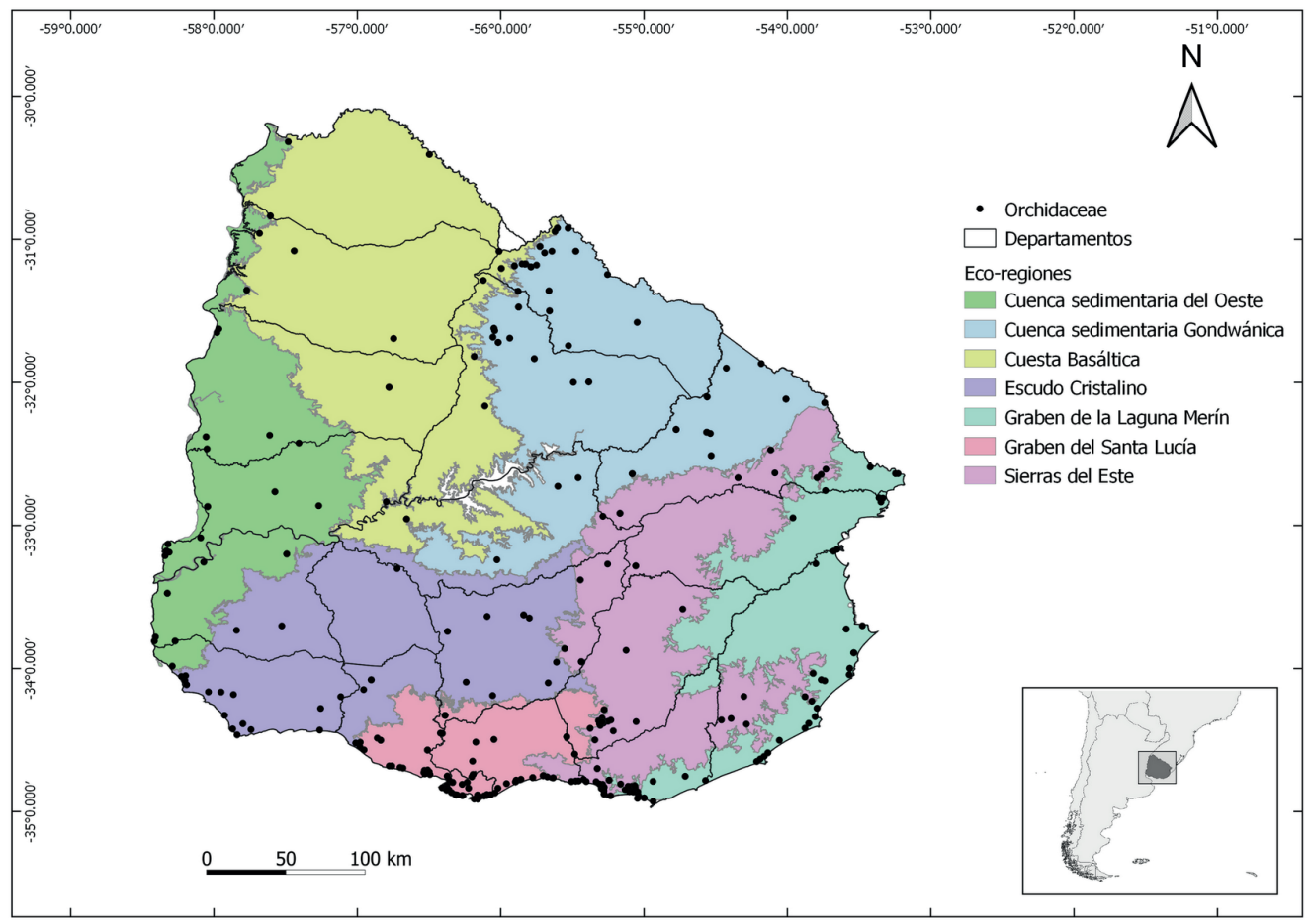

FiguRA 11. Distribución de Orchidaceae en eco-regiones de Uruguay. Mapa elaborado por L. Marín [departamentos: MVOTMA-Geoservicios. (2020a); eco-regiones adaptado de Brazeiro et al. (2015a)]. 


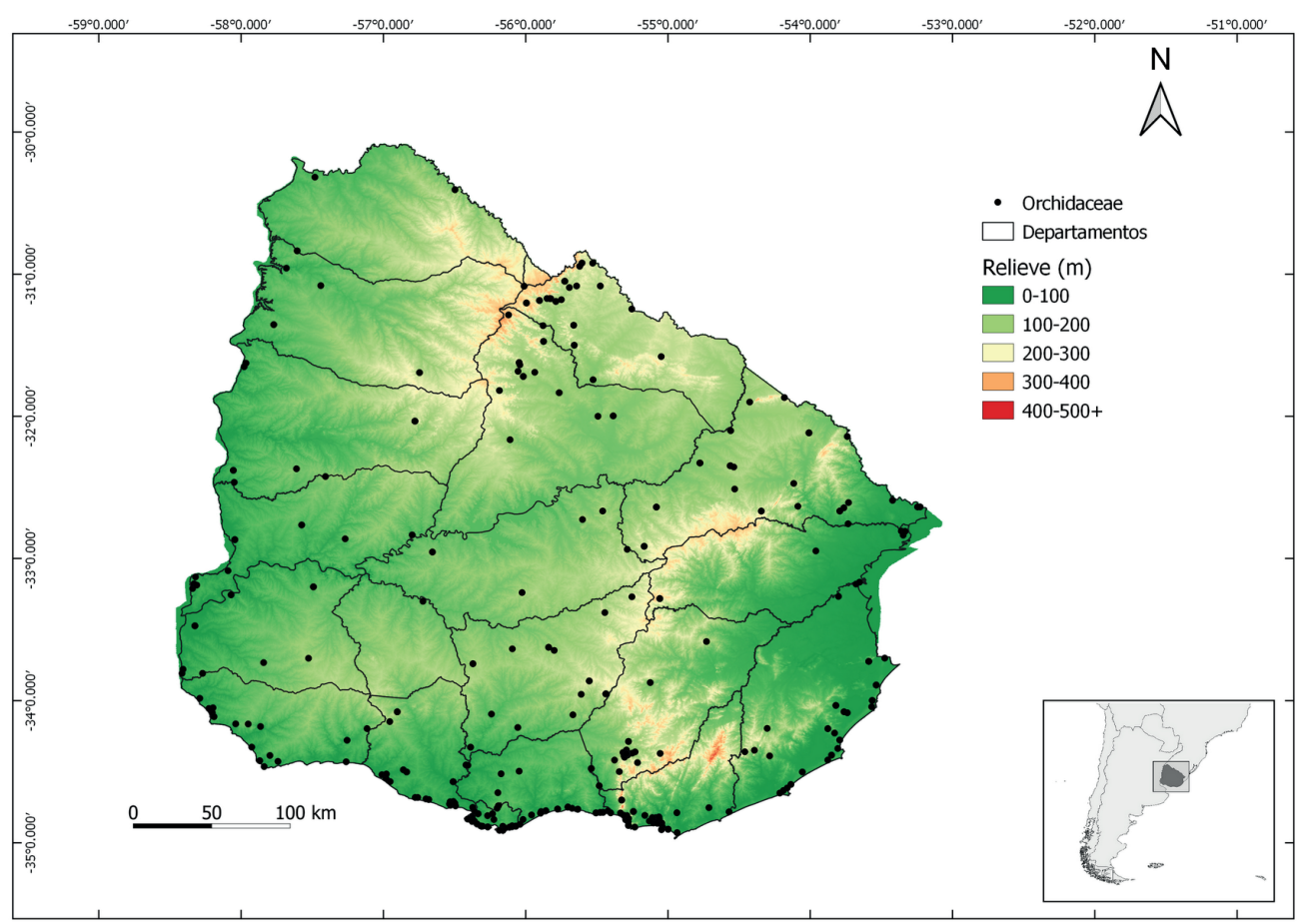

FiguRA 12. Distribución de Orchidaceae en relación al relieve del país. Mapa elaborado por L. Marín [departamentos: MVOTMA-Geoservicios. (2020a)].

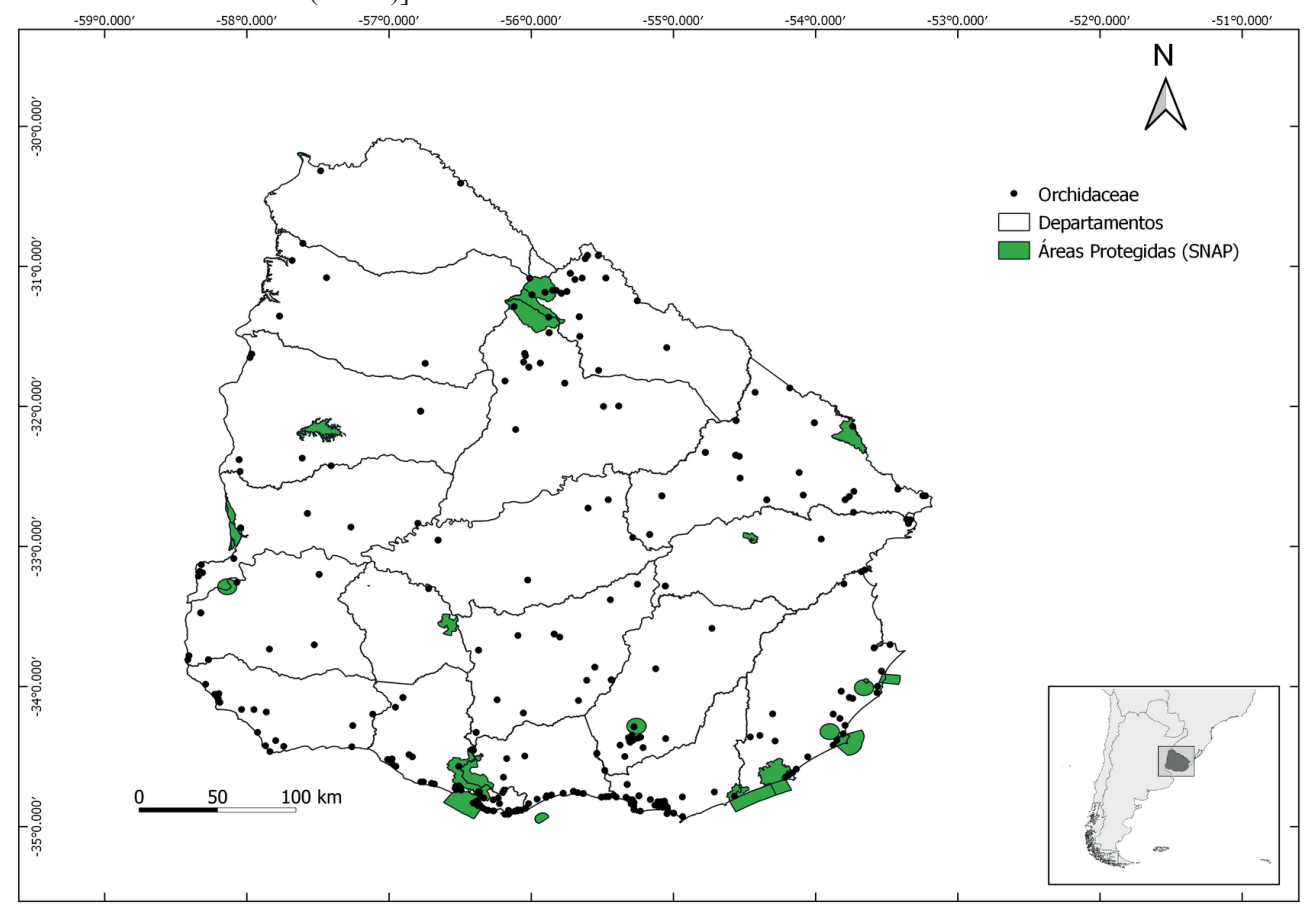

FiguRA 13. Distribución de Orchidaceae en departamentos de Uruguay en relación al Sistema Nacional de Áreas Protegidas (SNAP). Mapa elaborado por L. Marín [departamentos: MVOTMA-Geoservicios. (2020a); áreas de conservación adaptado de SNAP (2020)]. 
de extinción (Brazeiro et al. 2008). Se destacan los ambientes de BS y BQ de los departamentos TAC y RIV, en los que se encuentra la mayoría (14 spp.) de las especies amenazadas de la región.

Por último, cerca del $20 \%$ de las especies del país presentan una única o pocas colectas (entre una y tres) y $30 \%$ tienen registros de colectas a inicios del siglo pasado y en áreas que se han convertido en zonas urbanizadas. Varias de estas (Brachystele waldemarii. Habenaria platanthera y Pelexia arechavaletae), son endémicas del país y solo se conocen por el espécimen typus ya que no han sido colectadas nuevamente, por lo que existe la posibilidad que puedan haberse extinguido con el paso del tiempo y por eso no existe hasta la fecha registros científicos ni muestras adicionales en los herbarios. Esto puede influir en la disminución de la riqueza de especie de 59 a 43 especies para el país, por lo que es preciso priorizar el enfoque y esfuerzo de colecta hacia estas especies que podrían estar extintas en el país.

\section{INVENTARIO DE ESPECIES}

ACIANTHERA Scheidw.

1. Acianthera hygrophila (Barb.Rodr.) Pridgeon \& M.W.Chase, Lindleyana 16 (4): 244 (2001).

Pleurothallis hygrophila Barb.Rodr., Gen. Spec. Orchid. 1: 7 (1877).

Arthrosia hygrophila (Barb.Rodr.) Luer, Monogr. Syst. Bot. Missouri Bot. Gard. 105: 249 (2006).

Specklinia hygrophyla (Barb.Rodr.) F. Barros, Hoehnea 10: 110 (1983 publ. 1984).

Stelis hygrophila (Barb.Rodr.) Pridgeon \& M.W. Chase, Lindleyana 16 (4): 263 (2001).

DistRIBUCIÓN: Brasil, Argentina, Uruguay (CER).

AMBIENTE: BR.

ECO-REGIÓN: CuSeGo.

HÁBIto: Epífita.

EstADO DE CONSERVACIÓN: No consta en Marchesi et al. (2013). Cumple con los criterio 1, 2, se sugiere adicionar a la lista de especies prioritarias para el país. Material examinado: URUguay. Cerro Largo, Río Yaguarón, Paraje La Gloria, 22-IX-2013, Rossado et al. 213 (MVJB 29163); idem, cultivada en Montevideo, 22-II2013, Rossado \& Mai 178 (MVJB 29164).

NotA: Registrada por Rossado et al. (2014) en el Noreste del país ampliando su límite de ocurrencia hacia el sur. Hasta el momento se reporta únicamente en una localidad.

2. Acianthera pubescens (Lindl.) Pridgeon \& M.W.Chase, Lindleyana 16 (4): 245-246 (2001).

Pleurothallis pubescens Lindl., Companion Bot. Mag. 2: 355 (1836).

Humboltia pubescens (Lindl.) Kuntze, Revis. Gen. P1. 2: 668 (1891).

Distribución: Argentina, Bolivia, Brasil, Colombia,
Ecuador, Guayana Francesa, México, Paraguay, Perú, Surinam, Uruguay (CER), Venezuela.

Ambientes: BR, BQ, BS, $\mathrm{H}$.

ECO-REGiONES: CuSeGo.

НÁBito: Epífita.

EstADO DE CONSERVACIÓN: Prioritaria (Criterios 3,4).

Material examinado: URUguay. Cerro Largo, Cuchilla Cambotá, Establecimiento Santa Sofía, 15-XII2014, Brussa et al. 29192 (MVJB); Cuchilla Verde, Río Branco, 12-XII-2002, Izaguirre s.n. (MVFA 32857); 08-I1980, Brescia s.n. (MVFA 16348).

Nota: Izaguirre (2013) describe su presencia en el Noreste del país.

3. Acianthera sonderiana (Rchb.f.) Pridgeon \& M.W.Chase, Lindleyana 16 (4): 246 (2001).

Pleurothallis sonderiana Rchb.f., Linnaea 22: 830 (1850).

Humboltia sonderiana (Rchb.f.) Kuntze, Revis. Gen. Pl. 2: 668 (1891).

Specklinia saundersiana (Rchb.f.) F.Barros, Hoehnea 10: 110 (1984).

DistribuCIÓn: Argentina, Brasil, Paraguay, Uruguay (CER).

AmbIentes: BQ, BR, BS.

ECO-REGIÓN: CuSeGo.

HÁBıтo: Epífita

Estado de CONSERVACIÓn: Prioritaria (Criterio 3).

Material examinado: Uruguay. Cerro Largo, Cuchilla Cambotá, Establecimiento Santa Sofía, 15-XII2014, Brussa et al.s.n (MVJB 29191), Cañada de la Isla larga, 08-X-2014, Brussa \& Alvarez s.n. (MVJB 29498), Quebrada de los Cuervos, 01-XI-2000, Altez s.n (MVFA 32149), Arroyo Sarandí, Sierra de Ríos, 15-V-1999, Brussa \& Grela s.n. (MVFA 29143), Sierra de Ríos, 21-X-1992, Izaguirre et al. s.n. (MVFA 21093B). 
Nota: Izaguirre (2013) describe su presencia en el Noreste del país.

\section{Bipinnula Comm. ex Juss.}

4. Bipinnula biplumata (L.f.) Rchb.f., Xenia Orchid. 3: 62 (1883).

Arethusa biplumata L.f., Suppl. Pl.: 405 (1782).

Bipinnula bonariensis Spreng., Syst. Veg. 3: 745 (1826), nom. illeg.

DistriBución: Brasil, Argentina, Uruguay (MON).

AMBIENTE: P.

ECO-REGIÓN: GrSaLu.

HÁBITO: Terrestre.

Estado de Conservación: Prioritaria (Criterios 1, 2, 3, 4). Material examinado: URUGUAY. Montevideo, Parque Lecoq, 21-XII-1977, Brescia s.n (MVFA 15131); en campo, XI/1877, Arechavaleta 2628 [(MVFA) Citada en Izaguirre (1973), No vista].

Nota: Descrita en el país por Izaguirre (1973) indicando su distribución en pocas localidades de Montevideo.

5. Bipinnula gibertii Rchb.f, Linnaea 41: 51 (1876).

Distribución: Brasil, Uruguay (CER, MAL, MON, RIV).

Ambiente: BS, BQ, P.

ECo-Regiones: GrSaLu, CuSeGo, GrLaMe.

HÁBITo: Terrestre.

Estado de Conservación: Prioritaria (Criterio 1).

Material examinado: Uruguay. Cerro Largo, Río Negro, XII-1937, Gallinal et al. 1897 (MVM). Maldonado, Punta Ballena, 22-XI-1922, Osten 16875 (MVM). Montevideo, Punta Brava, "In campis", X-1867, Gibert 487 (MVFA); Cerro de Montevideo, 25-X-1983, Gago et al. s.n. (MVJB 22029). Rivera, Masoller, 1-IX-1969, Del Puerto s.n. (MVFA 2748).

NotA: Indicada para el país por Pabst (1952) e Izaguirre (1973) en áreas de suelos rocosos.

6. Bipinnula montana Arechav., Anales Mus. Nac. Montevideo 12: 282 (1899).

Bipinnula ctenopetala Schltr., Repert. Spec. Nov.

Regni Veg. Beih. 35: 24 (1925).

Bipinnula canisii Dutra ex Pabst, Arq. Bot. Estado

São Paulo, n.s., f.m., 3: 109 (1955).

DistribuCión: Brasil, Uruguay (CAN, LAV, MAL, RIV).

Ambientes: BS, BQ, P.

ECo-Regiones: CuSeGo, SiEs.

НА́вІто:Terrestre.
Estado de Conservación: Prioritaria (Criterio 1).

Material examinado: URUGUay. Canelones, Suárez, X-1945, Lombardo 5877 (MVJB) Citada en Izaguirre (1973), no visto]. Lavalleja, Cerro Arequita, 17-X-2010, Morrone 6323 (SI). Maldonado, Piriápolis, Cerro San Antonio, 28-IX-2003, Cirillo s.n. (MVFA 32848). Rivera, entre Masoller y Rivera, 1-IX-1969, Del Puerto \& Marchesi s.n. (MVFA 2749).

NotA: Izaguirre (1973) menciona su distribución en el país.

7. Bipinnula penicillata (Rchb.f.) Cisternas \& Salazar, Phytotaxa 64: 10 (2012).

Chloraea penicillata Rchb.f., Otia Bot. Hamburg.: 51 (1878).

Geoblasta penicillata (Rchb.f.) Hoehne ex M.N.Correa, Revista Mus. La Plata, Secc. Bot., n.s., 11 (54): 71 (1968).

Distribución: Argentina, Brasil, Uruguay (CAN, COL, LAV, MAL, MON, RIO, SAN, SOR, TAC). Ambientes: BR, BS, P.

Eco-regiones: CuSeOe, SiEs, GrSaLu, EsCr, CuSeGo.

HÁBito: Terrestre

EstAdo de CONSERVACIÓN: No consta en Marchesi et al. (2013).

Material examinado: URUGUAY. Canelones, Cuchilla Alta, 16-X-1949, Rosengurtt B5660 (MVM). Colonia, Riachuelo, 11-X-1936, Cabrera 3812 (SI). Lavalleja, Cerro del Verdún. 20-X-1962, Rosengurtt \& Del Puerto 8665 (MVFA). Maldonado, Cerro San Antonio, Piriápolis, 15-X-2002, Cirillo s.n. (MVFA 32844). Montevideo, Playa La Colorada, 12-X-1986, Crosa s.n. (MVFA 18459). Río Negro, Río Uruguay, 22-X-1942, Rosengurtt B4097 (MVM). San José, Sierra de Mahoma, 21-X-1966, Izaguirre et al. s.n. (MVFA 2602). Soriano, Vera, 25-X-1898, Berro 435 (MVFA). Tacuarembó, Unidad Experimental La Magnolia, 11-X-1991, Bayce et al. s.n. (MVFA 22146).

8. Bipinnula polysyka Kraenzl., Bot. Jahrb. Syst. 9: 317 (1887).

DistriBuCIÓn: Argentina, Uruguay (CAN, COL, LAV, MAL, MON, PAY, RIO, SAN, SOR).

AMBIENTES: P, BS.

ECo-REgiones: GrSaLu, SiEs, EsCr.

HÁBIto: Terrestre.

Estado de Conservación: Prioritaria (Criterio 1).

Material examinado: URUGUay. Canelones, Independencia, 18-XI-1898 Berro 319, Osten 4870 (MVM). 
Colonia, Ruta 22 entre Minas de Narancio y Tarariras, 1-XI-1999, Kiesling 9315 (SI). Lavalleja, Minas Orientales Ruta 60, 19-XI-1969, Supino \& Cabrera s.n. (MVFA 2753). Maldonado, Sierra Ballena, Cerros al lado de Laguna del Sauce, 22-XI-1971, Lombardo et al. s.n. (MVFA 10973). Montevideo, Parque Lecocq Camino Azarola, 8-XI-2001, Masciadri s.n. (MVJB 23547). Paysandú, Pandule, 15XI-1991, Marchesi et al. s.n. (MVFA 20686). Río Negro, Tres Bocas Ruta 24 y 15, 14-XI-1991 Marchesi s.n. (MVFA 20683). San José, Sierra de Mahoma, XI-1968, Izaguirre et al. 7820 (MVFA citado en Izaguirre (1973) pero no localizado). Soriano, Cerro Vera, 4-X-1900, Berro 1319 (MVFA).

NotA: Indicada en Pabst (1952) e Izaguirre (1973).

\section{BRACHYSTELE Schltr.}

9. Brachystele arechavaletae (Kraenzl.) Schltr., Beih. Bot. Centralbl. 37(2): 372 (1920).

Spiranthes arechavaletae Kraenzl., Bot. Jahrb. Syst. 36 (80): 9 (1905).

Diskyphogyne arechavaletae (Kraenzl.) Szlach. \& R.González, Fragm. Florist. Geobot. 41: 495 (1996).

DistribuCIÓN: Brasil, Uruguay (CAN, COL, FLI, ROC, TAC).

Ambientes: BR, P.

ECO-REgiones: EsCr, CuSeOe, GrLaMe, GrSaLu. HÁBito: Terrestre.

Estado de CONSERVACión: Prioritaria (Criterios 1,3).

Material examinado: URUguay. Canelones, Guazubira, 15-XII-1994, Rabaiotti et al. s.n. (MVFA 24314). Colonia, Conchillas, 01-XI-1969, Del Puerto \& Marchesi s.n (MVFA 9098). Florida, Timote, IV-1937, Gallinal et al. B1638 (MVFA). Rocha, Estación Biológica Potrerillo de Santa Teresa, 18-XII-2003, Beyhaut \& Bayce s.n. (MVFA 32957). Tacuarembó, XI-1898, Arechavaleta 10 (HBG).

Nota: Indicada como endémica del país en Schinini (2010), con distribución restringida en Uruguay y al sur de Brasil (Flora del Cono Sur). Mencionada entre las especies de Diskyphogyne (Szlachetko \& Tamayo 1996).

10. Brachystele camporum (Lindl.) Schltr., Beih. Bot. Centralbl. 37(3): 372 (1920).

Spiranthes camporum Lindl., Gen. Sp. Orchid. Pl.: 473 (1840).

Gyrostachys camporum (Lindl.) Kuntze, Revis. Gen. P1. 2: 664 (1891).

Brachystele delicatula auct. non (Kraenzl.) Schltr.
Spiranthes delicatulaKraenzl., Bot. Jahrb. Syst. 36(80): 9 (1905)

DistribuCiÓn: Argentina, Brasil, Paraguay, Uruguay (CAN, MAL, MON, LAV, PAY, RIO, RIV, ROC, SAN, SOR, TRE).

AMBIENTES: BR, BS, P.

Eco-regiones: GrSaLu, SiEs, GrLaMe, CuSeGo, $\mathrm{CuSeOe}$.

HÁBIto: Terrestre.

Estado DE CONSERVACión: No consta en Marchesi et al. (2013).

Material examinado: URUguay. Canelones, Santa Lucía, Tweedie 536 (K). Maldonado, Punta Ballena, 30XII-2018, Marín et al. OR2 (MVJB). Montevideo, Parque RIVera 10-II-2005, Aretche 18 (MVJB) Lavalleja, Aigua, 20-IV-1935, Legrand 590 (MVM). Paysandú, Estación Experimental Mario Cassinoni 16-III-1988, S.leg, s.n. (MVFA 19499). Río Negro, Fray Bentos, 22-XI-1913, Berro 7171 (MVFA). Rivera, Gruta de Piria, Cuchilla Negra, 15-XI-1996, Bonifacino. et al. s.n. (MVFA 26018). Rocha, Valizas, 03-II-2004, Callero. s.n. (MVJB 22462). San José, Sierra de Mahoma, 04-XII-2012, Sanguinetti 66 (SI). Soriano, Vera, 10-X-1900, Berro 1316 (MVFA). Treinta y Tres, Río Tacuarí, Paso del Dragón, 08-I-1980, Brescia et al. s.n. (MVFA 16313).

Nota: Ocurrencia indicada en Pabst (1952). Afín a B. dilatata según Schinini (2010) y Correa (1955) diferenciada en este último, por las brácteas delicadas y mas cortas que las flores ( $v s$. brácteas tiesas, agudas y mas largas que las flores).

11. Brachystele cyclochila (Kraenzl.) Schltr., Beih. Bot. Centralbl. 37(3): 373 (1920).

Spiranthes cyclochila Kraenzl., Kongl. Svenska Vetensk. Acad. Handl., n.s., 46(10): 36 (1911).

Brachystele cycloglossa Schltr, Repert. Spec. Nov. Regni Veg. 16: 321 (1920).

DistribuCión: Argentina, Brasil, Paraguay, Uruguay (MAL, SOR).

Ambientes: BS, BC.

ECo-Regiones: GrLaMe, SiEs, CuSeOe.

HÁBIto: Terrestre.

ESTADO DE CONSERVACIÓN: No consta en Marchesi et al. (2013).

Material Examinado: URUguay. Soriano, Cerro de la Cueva del Tigre, 03-I-1903, Berro 3075 (MVFA).

NotA: Indicada para el país en Pabst (1952).

12. Brachystele dilatata (Lindl.) Schltr., Beih. Bot. Centralbl. 37(3): 373 (1920). 
Spiranthes dilatata Lindl., Gen. Sp. Orchid. Pl.: 474 (1840).

Gyrostachys dilatata (Lindl.) Kuntze, Revis. Gen. P1. 2: 664 (1891).

Spiranthes ulaei Cogn.,Fl. Bras. 3(4): 207 (1895).

Brachystele ulaei (Cogn.) Schltr., Beih. Bot. Centralbl. 37(3): 374 (1920).

Brachystele hoehnei Pabst, Bradea 1(8): 53 (1971).

Distribución: Argentina, Brasil, Paraguay, Perú, Uruguay (CAN, LAV, MAL, MON, RIO, RIV, ROC, SAN, TAC).

Ambientes: BC, BS, BR, $\mathrm{H}$.

Eco-Regiones: GrSaLu, CuSeGo, SiEs, GrLaMe, EsCr. HÁBIto: Terrestre.

ESTADO DE CONSERVACIÓN: No consta en Marchesi et al. (2013).

Material examinado: URUGUAY. Canelones, Cuchilla Alta, 08-XII-2013, Haretche 700 (MVJB). Lavalleja, Cerro Guazubirá, 30-XII-1951, Teague s.n. (MVM 15235). Maldonado, Pueblo Eden, 25-I-2019, Marin et al. OR22 (MVJB); Punta Negra, 29-II-2019, Marin \& Perez OR19 (MVJB).. Montevideo, Punta Espinillo, 04II-1981, Izaguirre \& Marchesi s.n. (MVFA 16808). Río Negro,Estancia La Tuna Blanca (Echevarne), 27-I-1999, Marchesi \& Vignale s.n. (MVFA 28738). Rivera, Camino Minuano, Masolles, 02-II-1958 Rosengurtt B7154 (MVFA). Rocha, Cerro del Indio, 15-I-1958, Zorrón 6 (MVHC 1292). San José, Kiyú, 15-I-1970, Izaguirre 2761 (MVFA). Tacuarembó, Estancia La California, 30-I-1962, Millot \& Del Puerto 1128 (MVFA).

NotA: Indicado para el país por Pabst (1952)

13. Brachystele pappulosa Szlach., Fragm. Florist. Geobot. 41(2): 848-849 (1996).

DistribuCión: Uruguay (MON). Endémica del país. ECOREGIÓN: GrSaLu.

HÁBIto: Terrestre.

EstAdO DE CONSERVACIÓN: No consta en Marchesi et al. (2013). Cumple con los criterio 1, 2, 3, se sugiere adicionar a la lista de especies prioritarias para el país. Material examinado: URUGUAY. Montevideo, Banda Oriental, 1816-1821, S. Hilaire C22406 (P Photo).

14. Brachystele waldemarii Szlach., Fragm. Florist. Geobot. 41(2): 850-851 (1996).

Distribución: Uruguay (LAV). Endémica del país.

AmBientes: BS.

ECO-REGIÓN: SiEs.

HÁBIто: Terrestre.
Estado de CONSERVACIÓN: No consta en Marchesi et al. (2013). Cumple con los criterios 1, 2, 3, se sugiere adicionar a la lista de especies prioritarias para el país. Material Examinado: URUGUAY. Lavalleja, Minas, III1918, Arechavaleta s.n. (HBG 524499 Photo).

Nota: Especie posiblemente extinta, o muy rara. Descrita junta con B. pappulosa por Szlachetko (1996), ambas sin registros de colectas posteriores en los herbarios del país.

\section{Campylocentrum Benth.}

15. Campylocentrum densiflorum Cogn., Fl. Bras. 3(6): 511 (1906).

Campylocentrum dutrai Schltr., Repert. Spec. Nov. Regni Veg. Beih. 35: 108 (1925).

Campylocentrum hasslerianum Hoehne, Arq. Bot. Estado Sao Paulo 1(1): 23(1938).

Campylocentrum hatschbachii Schltr., Repert. Spec. Nov. Regni Veg. 23: 70(1926).

DistribuCión: Argentina, Brasil, Uruguay (CER, TRE). AmBientes: BS, BR.

ECO-REgIONES: CuSeGo, $\mathrm{CuBa}$.

HÁBITo: Epífita.

Estado De CONSERVACión: Prioritaria (Criterio 3).

Material examinado: URUGUAY. Cerro Largo, Cañada de Santos, Establecimiento Techera, 08-XII-2016, Brussa s.n. (MVJB 30406). Treinta y Tres, RIO Tacuarí, 11-XII2014, Rossado 413 (MVJB 30358).

NotA: Andrade et al. (2018) y Mai et al. (2019) mencionan el registro de $C$. aromaticum [MVFA 19156] sin embargo Pessoa \& Alves (2019) indican que esta especie es sinonimo de C. brachycarpum y endémica del bosque Atlántico del Sur y Sureste de Brasil siendo confundida con C. densiflorum.

\section{CAPANEmia Barb.Rodr.}

16. Capanemia micromera Barb.Rodr., Gen. Spec. Orchid. 1: 138 (1877).

Quekettia micromera (Barb.Rodr.) Cogn., Fl. Bras. 3(6): 198 (1904).

Distribución: Argentina, Bolivia, Brasil, Paraguay, Uruguay (CER, RIV, ROC, TAC, TRE).

AMBIENTES: BS, BPa, BR.

ECo-REgiones: CuSeGo, CuBa, GrLaMe.

HÁBITo: Epífita.

Estado de CONSERVACIÓN: Prioritaria (Criterio 3, 4). 
Material examinado: URUGUay. Cerro Largo, Sierra Aceguá, 16-XI-1987, Izaguirre et al. s.n. (MVFA 19099). Rivera, Arroyo Laureles, 2006, Brussa s.n. (MVJB 25152). Rocha,Estancia el Palmar, 18-IX-1979, Izaguirre s.n. (MVFA 16832). Tacuarembó, Cañada de Lucio, Establecimiento Santa María de Zamora, 24-IX-2016, Brussa s.n. (MVJB 30301). Treinta y Tres, Arroyo Parao, Vergara, 1963, Romano 4142 [(MVJB) Citado en Izaguirre (2013), no visto].

NotA: Izaguirre (1972, 2013) у Mai et al. (2019) menciona su distribución en el país y Buzzato et al.(2020) indica la presencia en el sureste y noreste.

17. Capanemia superflua (Rchb.f.) Garay, Bot. Mus. Leafl. 21: 261 (1967).

Oncidium superfluum Rchb.f., Ann. Bot. Syst. 6: 721 (1863).

Capanemia superflua (Rchb.f.) Garay var. rosea Izag., Agro. 17:26 (2013).

Capanemia uliginosa Barb.Rodr., Gen. Spec. Orchid. 1: 137 (1877).

Rodriguezia anomala Rolfe, Gard. Chron., ser. 3, 9: 728 (1891).

Rodriguezia juergensiana Kraenzl., Notizbl.

Königl. Bot. Gart. Berlin 2: 377 (1895).

Rodriguezia uliginosa (Barb.Rodr.) Cogn., Fl.

Bras. 3(6): 169 (1904).

Distribución: Argentina, Brasil, Uruguay (CER).

AMBiente: BR, BPa.

ECO-REGIÓN: CuSeGo.

НÁBIto: Epífita

Estado DE CONSERVACión: Prioritaria (Criterio 3).

Material examinado: URUGUAY. Cerro Largo, río Yaguarón, 18-VII-2008, Berazategui \& Duarte s.n. (MVM 21147 no visto); Estancia La Tigra, arroyo Yerbalito, 17VII-2008, Duarte \& Berazategui s.n. [(MVM 21160) Citado en Izaguirre (2013), no visto].

Nota: Izaguirre (2013) y Mai et al. (2019) indican su distribución en el país y de Capanemia superflua (Rchb.f.) Garay var. rosea posteriormente sinonimizada por Buzzato et al. (2020).

\section{Chloraea Lindl.}

18. Chloraea bella Hauman Anales de la Sociedad Científica Argentina 90: 99 (1920)

DisTRIBUCIÓN: Argentina, Uruguay (RIO).

HÁBIто: Terrestre

Estado de Conservación: Prioritaria (Criterio 1, 3).
Material eXAminado: No localizado.

Nota: Citada para Uruguay en la Flora del Cono Sur (2020), en Buzatto et al. (2014) como endémica de Entre Ríos y áreas vecinas de Uruguay y como especie prioritaria para conservación en el país por Marchesi et al. (2013). Correa (1969) indica que junto con $C$. membranaceae son las únicas especies de Chloraea que ocurren fuera de la zona andina, sin embargo indica que $C$. bella solo fue colectada en la provincia de Entre Ríos (Argentina). En los herbario del país no fueron encontrados registros.

19. Chloraea membranacea Lindl., Gen. Sp. Orchid. P1.: 401 (1840).

Chloraea membranacea var. paranaensis Schltr., Repert. Spec. Nov. Regni Veg. 23: 33 (1926).

Distribución: Argentina, Brasil, Uruguay (CAN, CER, COL, FLI, MAL, MON, RIO, SAN, SOR, TAC, TRE). Ambientes: BR, BS, BP, BC, P.

Eco-regiones: GrSaLu, EsCr, $\mathrm{CuSeOe}, \mathrm{CuSeGo.}$

HÁBITO: Terrestre.

ESTADO DE CONSERVACIÓN: No consta en Marchesi et al. (2013).

Material examinado: URUGUay. Canelones, Ruta Interbalnearia, 27-XI-1967, Izaguirre 1980 (MVFA). Cerro Largo, Paso de Tía Lucía, 11-XI-1965, Izaguirre et al. 2437 (MVFA). Colonia,Arroyo San Pedro, 26XI-1972, Izaguirre \& Laguardia s.n. (MVFA 11826). Florida, Casupá, 22-XI-1969, Del Puerto 2754 (MVFA). Maldonado, Punta del Este, 22-XI-1947, Teague s.n. (MVM 15331). Montevideo, Parque Lecocq, Camino Azarola, 08-XI-2001, Radesca s.n. (MVJB 23218). Río Negro, Arroyo de Patos, 22-X-1942, Rosengurtt B4046 (MVM). San José, Kiyú, 10-XI-1972, Izaguirre et al. s.n. (MVFA 10914). Soriano, Parque Municipal 33 Orientales, XI-2010, Morrone 6340 (SI). Tacuarembó, Tacuarembó, XI-1898, Arechavaleta s.n. (MVFQ 171). Treinta y Tres, Río Tacuarí Ruta 8, 09-XI-1965, Del Puerto et al. s.n. (MVFA 2313).

NotA: Citada para el país en Pabst (1952).

\section{Cyclopogon C.Pres1}

20. Cyclopogon apricus (Lindl.) Schltr., Beih. Bot. Centralbl. 37(3): 384 (1920).

Spiranthes aprica Lindl., Gen. Sp. Orchid. P1.: 469 (1840).

Gyrostachys aprica (Lindl.) Kuntze, Revis. Gen. Pl. 2: 664 (1891). 
Beadlea aprica (Lindl.) Garay, Bot. Mus. Leafl. 28(4): 299 (1982).

Warscaea aprica (Lindl.) Szlach., Fragm. Florist. Geobot. 39(2): 561 (1994)

Cyclopogon paludosus (Cogn.) Schltr. Beih. Bot.

Centralbl., Abt. 2 37(3): 392 (1920)

Cyclopogon diversifolium (Cogn.) Schltr., Beih. Bot. Centralbl. 37(3): 387 (1920).

Beadlea diversifolia (Cogn.) Garay, Bot. Mus. Leafl. 28(4): 300 (1982).

Warscaea diversifolia (Cogn.) Szlach., Fragm. Florist. Geobot. 39(2): 562 (1994).

Warscaea platyunguis (Schltr.) Mytnik, Rutk. \& Szlach., Orchidee (Hamburg) 57: 577 (2006).

DistribuCión: Argentina, Bolivia, Brasil, Uruguay (ART, CAN, DUR, FLE, FLI, LAV, MAL, MON, RIV, ROC, TAC).

Ambientes: BR, BP, BC, BS, BQ, P, H.

Eco-regiones: CuSeOe, CuBa, CuSeGo, GrLaMe, SiEs, GrSaLu, EsCr.

HÁBIto: Terrestre.

Estado DE CONSERVACIÓN: No consta en Marchesi et al. (2013).

Material examinado: URUGUAy. Artigas, Cuareim, 18-IX-1902, Berro 2859 (MVFA). Canelones,Pedrera Ruta 11, 06-X-1957, Rosengurtt B6632 (MVFA). Durazno, Establecimiento La Paz, Arroyo Cordobal, 03-X-1961, Izaguirre 72 (MVFA). Flores, Río Yí y Arroyo Marincho, 10-IV-1937, Rosengurtt B1512 (MVFA). Florida, Campo experimental Los Olivos, 03-IX-1984, May s.n. (MVFA 17385); Arroyo Mansavillagra, 13-X-1946, Correa PE5691 (MVFA); Rio Yi, 21-IV-1943, Gallinal et al. PE5178 (MVM). Lavalleja, Salto del Penitente, 01-IV-1985, Fenis s.n. (MVFA 17497); Minas, 30-III-1907, Osten 4495 (MVM). Maldonado, Piriápolis, 31-III-1911, Osten 5521 (MVM). Montevideo,Colón, 20-IV-1902, Osten 4308 (MVM). Rivera, Cerro Mirriñaque, 20-XI-1986, Izaguirre et al. s.n. (MVFA 18433). Rocha, Parque San Miguel, 06X-1965, Del Puerto \& Marchesis.n. (MVFA 5266); Entrada Cabo Polonio R10 km261, 4-XI-1985, Alonso s.n. (MVFA 17979); Castillos, 22-IV-1943, Legrand 3309 (MVM). Tacuarembó, Ruta 31, 02-X-1959, Rosengurtt B7725 (MVFA).

NotA: Pabst (1952) indica su ditribución en el país.

21. Cyclopogon chloroleucus Barb.Rodr., Gen. Spec. Orchid. 1: iii (1877).

Spiranthes chloroleuca Barb.Rodr., Gen. Sp. Orchid. 1: 181. 1877.
Cyclopogon chloroleucus var. fontinalis Barb. Rodr., Gen. Spec. Orchid. 1(Index): iii (1877).

Cyclopogon chloroleucus var. longipetiolata Barb. Rodr., Gen. Spec. Orchid. 1(Index): iii (1877). Serapias polyaden Vell., Fl. Flumin. Icon. 9: t. 56. 1831 [Arq. Mus. Nac. Rio de Janeiro 5: 371. 1882]. Stigmatosema polyaden (Vell.) Garay in Bot. Mus. Leafl. 28(4):377. (1982).

Cyclopogon polyaden (Vell.) F.S.Rocha \& Waechter in Acta Bot. Brasil. 20: 78. (2006).

Distribución: Argentina, Brasil, Paraguay, Uruguay (CER, RIV, TAC).

AmbIentes: BR, BS, BQ.

ECo-Regiones: $\mathrm{CuBa}$, CuSeGo.

HÁBITO: Terrestre.

EstADO DE CONSERVACIÓN: Prioritaria (Criterio 3).

Material examinado: URUguay. Cerro Largo, Sierra de Ríos, 10-X-1999, Brussa \& Grela s.n. (MVFA 29362). Rivera, Arroyo La Aurora, Establecimiento San Juan, 25-IX-2004, Brussa \& Grela 1212 (MVJB 22023). Tacuarembó, XI-1905, Arechavaleta s.n. (MVFQ 178). Nota: La flora del Cono Sur (2020) coloca como sinónimo de Cyclopogon polyaden (Vell.) F.S.Rocha \& Waechter a Serapias polyaden Vell., C. chloroleucus Barb.Rodr., Spiranthes chloroleuca Barb.Rodr. y Stigmatosema polyaden (Vell.) Garay. Por su parte, Buzatto et al. (2013) consideran dudosa e insuficiente la evidencia para tal consideración y proponen la exclusión de Serapias polyaden.

22. Cyclopogon congestus (Vell.) Hoehne, Fl. Brasílica 8(12: 2): 209 (1945).

Serapias congesta Vell., Fl. Flumin. Icon. 9:54 (1831).

Spiranthes alpestris Barb.Rodr., Gen. \& Spec. Orch. 1: 184 (1877).

Spiranthes alpestris Barb.Rodr. var. bidentata Barb.Rodr. Gen. \& Spec. Orch. Nov. 2:285 (1882). Cyclopogon alpestris Barb.Rodr., Gen. \& Spec. Orch. 1: 3.1877.

Beadlea congesta (Vell) Garay, Bot. Mus. Leafl. 28(4): 300.1980.

Cyclopogon lanegei Schltr., Feddes Repert. Spec. Nov. Regni Veg. Beih. 16: 392.1920.

Distribución: Argentina, Brasil, Paraguay, Uruguay (CER, FLI, LAV, MAL, SAL).

AmbIentes: BS, BP, BQ.

ECo-REgiones: CuSeGo, SiEs, EsCr, CuBa. 
HÁBito: Terrestre.

ESTADO DE CONSERVACIÓN: No consta en Marchesi et al. (2013).

Material examinado: URUGUAY. Cerro Largo, Ruta 26 $\mathrm{km}$ 48,5, 17-XI-1987, Izaguirre et al. s.n. (MVFA 191136). Florida, Arroyo Casupá, 27-X-1968, Del Puerto \& Marchesi 7638 (MVFA). Lavalleja, Sierra de Carajé Ruta 60, 26-X-1958, Rosengurtt B7436 (MVFA). Maldonado, Cerro Pan de Azúcar, 01-XI-1968, Del Puerto \& Marchesi 7730 (MVFA). Salto, Arroyo Valentín Grande y Ruta 31, 30-VIII-1969, Del Puerto \& Marchesi 8607 (MVFA).

23. Cyclopogon elatus (Sw.) Schltr., Repert. Spec. Nov. Regni Veg. Beih. 6: 53 (1919).

Satyrium elatum Sw., Prodr. : 119 (1788).

Neottia elata (Sw.) Sw., Kongl. Vetensk. Acad. Nya Handl. 21: 226 (1800)

Ibidium elatum (Sw.) Salisb., Trans. Hort. Soc. London 1: 291 (1812).

Spiranthes elata (Sw.) Rich., De Orchid. Eur. Ann.: 37 (1817).

Sauroglossum richardii Ames, Orchidaceae 1: 44 (1905).

Beadlea elata (Sw.) Small, Brooklyn. Bot. Gard. Mem. 1: 38 (1918).

Distribución: Argentina, Brasil, Colombia, Guayana Francesa, Paraguay, Perú, Surinam, Uruguay (ART, CAN, CER, COL, FLI, LAV, MAL, RIO, RIV, ROC, SAL, SAN, SOR, TAC, TRE).

AMBIENTES: BP, BQ, BC, BR, BS.

Eco-regiones: CuSeOe, CuBa, CuSeGo, SiEs, GrLaMe, EsCr, GrSaLu.

HÁBITO: Terrestre.

ESTADO DE CONSERVACIÓN: No consta en Marchesi et al. (2013).

Material examinado: URUGUAy. Artigas, Estancia Silva y Rosa, Arroyo Mandiyú, 16-IX-1976, Marchesi s.n. (MVFA 12793). Canelones, Balneario Argentino, 27-X2005, Haretche 28 (MVJB 23429). Cerro Largo, Camino de Sierra de Río, Asperesas, 10-X-1999, Brussa \& Grela s.n. (MVFA 29357). Colonia, Playa Agraciada, 31-X1969, Del Puerto s.n. (MVFA 9099). Florida, Ruta 12 y Arroyo Arias, 18-X-1998, Bonifacino s.n. (MVFA 28752). Lavalleja, Cerro Arequita, 17-XII-1953, Rosengurtt \& Del Puerto 8346 (MVFA). Maldonado, Ruta 12 entre Pan de Azúcar y Minas, 10-X-1963, Arrillaga et al. 1618 (MVFA). Río Negro, Balneario Las Canas, X-2003, Cirillo s.n. (MVFA 32851). Rivera, Arroyo del Potrero, Campos de FYMNSA, 30-X-1997, Marchesi et al. s.n. (MVFA 27417). Rocha, Estación Biológica Potrerillo de Santa
Teresa, 28-XI-2002, Pérez s.n. (MVFA 32859). Salto, Arroyo Yacuí y Ruta 3, 09-IX-1978, Marchesi s.n. (MVFA 15746). San José, Sierra de Mahoma, X-2003, Cirillo s.n. (MVFA 32863). Soriano, Playa Agraciada, 26-XI-1972, Izaguirre \& Laguardia s.n. (MVFA 11882). Tacuarembó, Estancia La Loma, X-2002, Izaguirre s.n. (MVFA 32141). Treinta y Tres, Ruta 98, 10-VII-2003, Izaguirre \& Bayce s.n. (MVFA 32845).

Nota: Citada para el país en Pabst (1952), Correa (1955), Mai et al. (2019) y La Flora del Cono Sur (2020), especie con amplia distribución, posible confusión con Cyclopogon elegans Hoehne.

24. Cyclopogon longibracteatus (Barb.Rodr.) Schltr., Beih. Bot. Centralbl. 37(3): 390 (1920).

Spiranthes longibracteata Barb.Rodr., Gen. Spec. Orchid. 1: 185 (1877).

Beadlea longibracteata (Barb.Rodr.) Garay, Bot. Mus. Leafl. 28(4): 301 (1982).

Distribución: Argentina, Brasil, Paraguay, Uruguay (RIV).

AMBIENTE: BQ.

ECO-REGIÓN: CuBa.

HÁBIto: Terrestre.

Estado de CONSERVACiÓn: Prioritaria (Criterios 2,3).

Material Examinado: URUGUAY. Rivera, Bajada de Pena, 19-XI-1985, Marchesi s.n. (MVFA 17976); cultivada, origen Bajada de Pena, X-2003, Izaguirre s.n. (MVFA 32849).

25. Cyclopogon micranthus (Barb.Rodr.) Barb.Rodr., Gen. Sp. Orchid. 1 [Index]: iii. (1877).

Spiranthes micrantha Barb.Rodr., Gen. Sp. Orchid. 1: 183. (1877).

Hapalorchis lineata (Lindl.) Schltr., Beih. Bot. Centralbl. 37(3): 363 (1920).

Hapalorchis micrantha (Barb.Rodr.) Hoehne, Fl. Brasílica 8: 12(2): 291 (1945).

Distribución: Bolivia, Brasil, Guayana Francesa, Surinam, Uruguay (CAN, CER, DUR, ROC), Venezuela.

AMBIENTES: BR, BS, BC.

ECO-REGiones: GrSaLu, SiEs.

HÁBito: Terrestre.

ESTADO DE CONSERVACIÓN: No consta en Marchesi et al. (2013).

Material Examinado: URUGUAY. Canelones, Balneario Argentino, 27-X-2005, Haretche 27 (MVJB 23428); Arroyo La Coronilla Ruta interbalnearia km 70, 19-X- 
2003, Bonifacino s.n. (MVFA 32850). Cerro Largo, Sierra de Ríos, 04-X-2002, Brussa \& Grela s.n. (MVFA 32744). Durazno, Cuchilla de Pereira, 14-X-2006, Brussa \& Grela s.n. (MVJB 25281). Rocha, Cerro Rocha, 1998, Baez s.n. (MVFA 32154).

NотA: Especímenes identificados en los herbarios del país como $H$. lineata, sin embargo, este nombre es considerado sinónimo de $H$. micrantha por Govaerts et al. (2020), y de C. micranthus por Buzzato et al. (2013).

26. Cyclopogon oliganthus (Hoehne) Hoehne \& Schltr., Arch. Bot. São Paulo 1(3): 189 (1927).

Spiranthes oligantha Hoehne, (Revista Mus. Paul. Univ. São Paulo 10: 442 (1919).

Beadlea oligantha (Hoehne) Garay, Bot. Mus. Leafl. 28(4): 301 (1982).

Warscaea oligantha (Hoehne) Szlach., Fragm. Florist. Geobot. 39(2): 562 (1994).

DistriBuCión: Argentina, Brasil, Paraguay, Uruguay (LAV, RIO).

AMBIENTES: BR, BP.

ECO-REGIONES: CuSeOe, SiEs.

HÁBito: Terrestre.

ESTADO DE CONSERVACIÓN: No consta en Marchesi et al. (2013).

Material Examinado: URUGUAy. Lavalleja, Arroyo Gutierrez Ruta 8, 248,5 km, 15-IV-2010, Denhman 293 (SI). Río Negro, Balneario Las Cañas, X-2003, Cirillo s.n. (MVFA 32851).

27. Cyclopogon taquaremboensis (Barb.Rodr.) Schltr., Beih. Bot. Zentralbl. 37: 394 (1920).

Stenorrhynchos taquaremboense Barb.Rodr., Contr. Jard. Bot. Rio de Janeiro 1: 68 (1901).

Beadlea taquaremboensis (Barb.Rodr.) Garay, Bot.

Mus. Leafl. 28(4): 301 (1980 publ. 1982).

DistriBución: Brasil, Uruguay (LAV).

AMBIENTE: BS.

ECO-REGIÓN: GrSaLu.

HÁBITO: Terrestre.

ESTADO DE CONSERVACIÓN: No consta en Marchesi et al. (2013).

Material Examinado: URUGUAY. Lavalleja, Sierra de Minas, XI-1933. Mato 234 [MVM 10102 citado en Pabst (1952) no localizado]. Salto, Arapey, IX-1949, Herter 2405 (S No visto).

NotA: Su ocurrencia en Uruguay es citada en Andrade et al. (2019) y Pabst (1952) no obstante los registros no fueron localizados en los herbarios visitados. La flora del Cono Sur (2020) considera esta especie sinónimo de C. elatus con base en Correa (1955), sin embargo esta considera sinónimos unicamente a los especímenes Argentinos.

\section{Cyrtopodium R. Br.}

28. Cyrtopodium brandonianum Barb. Rodr., Gen. Sp. Orchid. 1: 132 (1877).

Cyrtopodium purpureum Rchb.f. \& Warm., Otia bot. Hamburg. 2:90 (1881)

Cyrtopodium gonzalezii L.C. Menezes,. Boletim CAOB 5(1) 9 (1995)

Distribución: Argentina, Bolivia, Brasil, Paraguay, DistRIBUCIÓN: Uruguay (RIV).

AmBIENTE: BQ.

ECO-REGIÓN: CuBa.

HÁBito: Rupícola.

Estado de Conservación: Prioritaria (Criterios 2, 3, 4). Material Examinado: URUGUAY. Rivera, Bajada de Pena, 22-XII-1992, Bayce et al. s.n (MVFA 21589); idem, 24-II-1992, Izaguirre et al. s.n. (MVFA 21463).

NotA: Izaguirre (2010) apunta su distribución en el país, la consideran una especies rara y con alta vulnerabilidad por perdida de ambientes de ocurrencia.

\section{GALEANDRA Lindl.}

29. Galeandra beyrichii Rchb.f., Linnaea 22: 854-855 (1850).

Galeandra viridis Barb.Rodr. Gen. Sp. Orchid. 2:176 (1881).

Galeandra fiebrigii Schltr., Repert. Spec. Nov. Regni Veg. Beih. 10:47 (1922).

Distribución: Argentina, Colombia, Bolivia, Brasil, Ecuador, Guayana Francesa, Paraguay, Perú, Surinam, Uruguay (ART, RIV), Venezuela.

AmbIentes: BQ, BS, BR

ECO-REGIÓN: CuBa.

HÁBIto: Terrestre.

ESTADO DE CONSERVACIÓN: Prioritaria (Criterio 3).

Material examinado: URUGUAY. Artigas: s.l., 22I-2002, Carbonell s.n. (MVFA 34846 No visto); lugar pedregoso dentro del monte, 10-I-2007, Carbonell s.n. (MVFA 34854 No visto). Rivera, Establecimiento Rinconada, 22-III-2013, Brussa s.n (MVJB 28599); Sierra de la Aurora, 22-VIII-2008, Brussa \& Malates s.n. (MVJB 26481); Arroyo Potrero, 12-XII-1997, 
Bonifacino et al. s.n. (MVFA 27640); Bajada de Pena, 29-II-1985, Marchesi et al. s.n. (MVFA 17631); Ruta 30, km 107, 11-IV-1984, Bayce et al. s.n. (MVFA 17321).

\section{GoMesA R.Br.}

30. Gomesa barbata (Lindl.) M.W.Chase \& N.H.Williams, Ann. Bot. (Oxford) 104 (3): 395 (2009).

Oncidium barbatum Lindl., Coll. Bot.: t. 23(1821). Alatiglossum barbatum (Lindl.) Baptista, Colet. Orquídeas Brasil. 3: 87. (2006.)

Oncidium suscephalum Barb.Rodr., Gen. Sp. Orchid. 2: 188 (1882).

DistRIBución: Bolivia, Brasil, Uruguay (CER).

AmbIEnTE: BQ.

ECO-REGIONES: SiEs.

НÁBıто: Epífita.

Estado de Conservación: Prioritaria (Criterio 3).

Material examinado: URUGUAY. Cerro Largo, Sierra de Ríos. 23-X-1991, Ziliani, Grun \& Baycé s.n. [(MVFA 20350) citado en Izaguirre (2013) No visto)]; Cuchilla Cambotá, 15-XII-2014, Brussa s.n. (MVJB 29206, 29188).

NотA: Indicada para el país en Izaguirre (2010) y Mai et al. (2019) .

31. Gomesa bifolia (Sims) M.W.Chase \& N.H.Williams, Ann. Bot. (Oxford) 104(3): 396 (2009).

Oncidium bifolium Sims, Bot. Mag. 36: t. 1491 (1812).

Oncidium bifolium var. majus hort. ex B.S.Williams, Orch.-Grow. Man., ed. 7: 383 (1894).

Coppensia bifolia (Sims) Dumort., (Mém. Acad. Roy. Sci. Belgique 9: 10 (1835).

Ampliglossum bifolium (Sims) Campacci, Colet. Orquídeas Brasil. 3: 83 (2006).

Distribución: Argentina, Bolivia, Brasil, Paraguay, Uruguay (CER, COL, DUR, MAL, RIO, ROC, SAL, SAN, SOR, TAC, TRE).

AMBIENTES: BR, BP, BS.

Eco-regiones: CuSeOe, CuSeGo, GrLaMe, SiEs.

НÁBito: Epífita

EstADO DE CONSERVACIÓN: No consta en Marchesi et al. (2013).

Material examinado: URUguay. Cerro Largo, Arroyo Zapallar, Estancia Nueva, 14-XII-1962, Legrand 4793 (MVFA). Colonia, La Paz, 24-XI-2018, Marin ORI, (MVJB). Durazno, Río Yi, 2003, Izaguirre s.n. (MVFA 32862). Maldonado, Sierra de las Ánimas, 19-XII-1963,
Rosengurtt \&Del Puerto 9694 (MVFA). Río Negro, Balneario Las Cañas, 21-XII-1965, Del Puerto \& Marchesi s.n. (MVFA 5618). Rocha, Ruta $16 \mathrm{~km} \mathrm{41,500,10-XII-}$ 1986, Armand et al. s.n.(MVFA 18844). Salto, Río Uruguay, 30-X-1978, Del Puerto \& Marchesi s.n. (MVFA15957). San José, Arazati, XI-1934, Rosa-Mato 644 (MVM 10084). Soriano, Vera, X-1898, Berro 167; Osten 4872 (MVFA, MVM). Tacuarembó, Río Negro, 4-IV-1920, Montoro 1022 (MVFA). Treinta y Tres, Treinta y Tres, 11-XII-1937, Legrand 4588 (MVM).

NotA: Pabst (1952) y Mai et al. (2019) indican su distribución en el país.

32. Gomesa uniflora (Booth ex Lind1.) M.W.Chase \& N.H.Williams, Ann. Bot. (Oxford) 104: 398 (2009).

Oncidium uniflorum Booth ex Lindl., Edwards's Bot. Reg. 29: t. 43 (1843).

Alatiglossum uniflorum (Booth ex Lind1.) Baptista, Colet. Orquídeas Brasil. 3: 89 (2006).

Kleberiella uniflora (Booth ex Lind1.) V.P.Castro \& Cath,Richardiana 6: 158 (2006).

Oncidium biflorum Barb.Rodr., Gen. Sp. Orchid. 2: 187(1882).

Distribución: Argentina, Brasil, Paraguay, Uruguay (CER).

AMBIENTE: BR.

ECO-REGIÓN: GrLaMe.

HÁBITo: Epífita.

Estado de Conservación: Prioritaria (Criterio 3).

Material examinado: URUGUAY. Cerro Largo, Río Yaguarón, Paso del Medio, 02-II-2018, Brussa s.n. (MVJB 30652).

Nota: Indicada para el país en Mai et al. (2019).

\section{HabenARIA Willd.}

33. Habenaria achalensis Kraenzl., Bot. Jahrb. Syst. 16(2): 133 (1893).

Habenaria angustiloba Lillo \& Hauman, An. Mus. Nac. Hist. Nac. Buenos Aires 29: 357 (1917). Habenaria achalensis var. angustifolia Kraenzl., Kongl. Svenska Vetensk. Acad. Handl., n.s., 46(10): 12 (1911).

Distribución: Argentina, Brasil, Paraguay, Uruguay (SAN).

AMBIENTE: BR.

ECO-REGIÓN: GrSaLu

HÁBIto: Terrestre.

Estado DE CONSERVACión: No consta en Marchesi et al. (2013). 
Material examinado: URUGUAY. San José, Barra de Santa Lucía, 10-I-1930, Osten 21825A (MVM).

NotA: Govaert et al. (2010) la consideran sinónimo de $H$. paiveana. La distribución en el país es citada en Pabst (1952) y Batista et al. (2011a).

34. Habenaria bractescens Lindl., Gen. Sp. Orchid. P1.: 308 (1835).

Kusibabella bractescens (Lindl.) Szlach., Richardiana 4: 59 (2004).

Habenaria sartor f. minor Cogn., Bull. Herb. Boissier, sér. 2, 3: 929 (1903).

Habenaria pontagrossensis Kraenzl., Kongl. Svenska Vetensk. Acad. Handl., n.s., 46(10): 6 (1911). Habenaria kleyi Schltr., Repert. Spec. Nov.

Regni Veg. Beih. 35: 20 (1925).

Distribución: Argentina, Bolivia, Brasil, Colombia, Paraguay, Uruguay (ART, CAN, CER, COL, FLI, LAV, MAL, RIV, TAC), Venezuela.

Ambientes: BR, BS, BP.

Eco-Regiones: CuBa, CuSeGo, GrSaLu, EsCr.

HÁBITO: Terrestre.

ESTADO DE CONSERVACIÓN: No consta en Marchesi et al. (2013).

Material examinado: URUGUAY. Artigas, Arroyo Sepulturas, I-2001, Carbonell s.n. (MVFA 32837). Canelones, El Pinar, 08-II-2004, Callero s.n. (MVJB 22463). Cerro Largo, Ruta 26, 9-I-1989, Brescia et al. s.n. (MVFA 16508). Colonia, Carmelo, 07-VIII-1964, Arrillaga et al. 1916 (MVFA). Florida, Arroyo Timote, 1941, Gallinal et al. PE4549 (MVM). Lavalleja, Cerro Gigante, 17-II-2017, Muñoz s.n. (MVJB 30603). Maldonado, 1920, Osten 15708 (MVM). Rivera, Arroyo Zapucay, 12-II-2006, Delfino s.n. (MVJB 22863). Tacuarembó, Laureles, 07-II1989, Paz 621 (MVM).

NotA: Pabst (1952) y Batista et al. (2011a) indican su distribución en el país.

35. Habenaria exaltata Barb.Rodr., Gen. Spc. Orchid. 1: 156 (1877).

Habenaria henscheniana var. densiflora Cogn. F1. Bras. 3(4): 85 (1893).

Habenaria sceptrum Schltr., Repert. Spec. Nov. Regni Veg. 16: 249 (1919).

Distribución: Brasil, Paraguay, Uruguay (TAC).

AMBIENTE: $\mathrm{H}$.

ECO-REGión: CuSeGo.

HÁBITo: Terrestre.

ESTADO DE CONSERVACIÓN: No consta en Marchesi et al. (2013).
Material eXAminAdo: URUGUAY. Tacuarembo, Laguna del General, I-1940, Legrand 2032 (MVM).

NotA: Mencionada para el país en Batista et al. (2011a) sugiriendo la sinonimia con $H$. sceptrum indicada en Pabst (1952).

36. Habenaria gourlieana Gillies ex Lindl., Gen. Sp. Orchid. P1.: 309 (1835).

Kusibabella gourlieana (Gillies ex Lindl.) Szlach., Richardiana 4: 59 (2004).

Habenaria fastor Warm, ex Hoehne, Vidensk. Meddel. Dansk Naturhist. Foren. Kjøbenhavn 90, t. 8 , f. 4.1884 .

Macrocentrum mendocinumPhil., Sert. Mendoc. Alt. 43 (1871).

Habenaria spegazziniana Kraenzl., Beibl. Bot. Jahrb. Syst. 80: 10 (1905).

Habenaria burkartiana Hoehne Ex Cabrera, Notas Mus. La Plata, Bot. 1: 226 (1936).

Kusibabella burkartiana (Hoehne) Szlach., Richardiana 4: 59 (2004).

Distribución: Argentina, Bolivia, Brasil, Paraguay, Uruguay (CAN, CER, COL, FLI, LAV, MAL, MON, RIO, RIV, ROC, SAL, SAN, SOR, TAC, TRE).

Ambientes: BP, BS, BQ, BC, BR, H, P.

Eco-Regiones: CuSeOe, CuBa, CuSeGo, SiEs, GrSaLu, GrLaMe, EsCr.

HÁBITO: Terrestre.

ESTADO DE CONSERVACIÓN: No consta en Marchesi et al. (2013).

Material examinado: URUGUAY. Canelones, Santa Lucía, 1885, Gibert 998 (K). Cerro Largo, Arevalo, 13-III-2015, Muñoz s.n. (MVJB 29678). Colonia, Colonia Suiza, 1910, Hicken 38 (SI 39918). Florida, Cerro Colorado, 16-II-1944, Gallinal et al. PE4549 (MVFA). Lavalleja, Minas, III-1921, Osten 15752 (MVM). Maldonado, Piriápolis, 28-I-1912, Osten 5774 (MVM). Montevideo, Carrasco, II-1875, Arechavaleta 2625 (MVM). Río Negro, Campo El Jabalí, 02-IV1998, Marchesi s.n. (MVFA 28161). Rivera,Tranqueras, 03-III-2009, Brussa s.n (MVJB 27535). Rocha, San Miguel, 26-II-1948, Teague s.n. (MVM 15178). Salto, San Antonio, 02-II-1937, Rosengurtt B1071 (MVFA). San José, Río Santa Lucía, 19-III-1931, Osten 22184 (MVM). Soriano, Juan Jackson, II-1941, Gallinal et al. PE4763 (MVFA). Tacuarembó, Quebrada de los Cuervos, 20-II-1987, Izaguirre et al. s.n. (MVFA 18704). Treinta y Tres, Establecimiento La Charqueada, 30-I2002, Izaguirre s.n. (MVFA 31358).

Nota: Su distribución en el país es indicada en Pabst (1952) y Batista et al. (2011a). 
37. Habenaria leucosantha Barb.Rodr., Gen. Spec. Orchid. 1: 151 (1877).

Habenaria leucosantha var. riograndensis Pabst, Rodriguésia 14(26): 47 (1951).

Habenaria berroana Barb.Rodr., Contr. Jard. Bot. Rio de Janeiro. 4: 97 (1907).

Habenaria leucosantha var. berroana (Barb.Rodr.)

Pabst, Rodriguésia 14(26): 46 (1951).

DistribuCión: Bolivia, Brasil, Paraguay, Uruguay (RIV).

AMBiente: $\mathrm{H}$.

ECO-REGión: CuSeGo.

HÁBITo: Terrestre.

Estado de Conservación: Prioritaria (Criterio 2, 3).

Material examinado: URUGUAY. Rivera, Curticeiras, 20-XII-1901, Berro 1890 (MVM).

Nota: Indicada en el país en Batista et al. (2011a).

38. Habenaria macronectar (Vell.) Hoehne, Bot. Jahrb. Syst. 68: 128 (1937).

Orchis macronectar Vell., Fl. Flumin. Icon 9: t. 45 (1831).

Kusibabella macronectar (Vell.) Szlach., Richardiana 4: 61 (2004).

Habenaria sartor Lindl. London J. Bot. 2:622 (1843).

Habenaria helodes Rchb.f., Linnaea 22: 813 (1850).

Habenaria dolichoceras Barb.Rodr., Gen. Spc. Orchid. 1: 153 (1877).

Habenaria sartoroides Schltr., Repert. Spec. Nov. Regni Veg. 16: 248 (1919).

Habenaria juergensii Schltr., Repert. Spec. Nov. Regni Veg. Beih. 35: 20 (1925).

DistRIBUCIÓN: Brasil, Paraguay, Uruguay (CER, SOR, RIO, TAC).

AMBIENTES: BR, BS.

Eco-regiones: CuSeGo, CuSeOe.

HÁBITO: Terrestre.

ESTADO DE CONSERVACIÓN: No consta en Marchesi et al. (2013).

Material Examinado: Uruguay. Cerro Largo, Palleros, I-1926, Osten 18370 (MVM). Río Negro, 20-II1908, Berro 4221 (MVM). Soriano, Vera, Berro C. 1472 (MVM). Tacuarembó, Rincón de la Laguna, 14-II-1947, Pabst s.n. (MVM 13904).

Nota: Pabst (1952) y Batista et al. (2011b) indican su distribución en el país.
39. Habenaria montevidensis Spreng., Syst. Veg. 3: 692 (1826).

Habenaria arechavaletae Kraenzl., Bot. Jahrb. Syst. 16(2): 185 (1892).

Habenaria arechavaletae var. elata Cogn.,Fl. Bras. 3(4): 92 (1893).

Habenaria obovatipetala Schltr., Repert. Spec. Nov. Regni Veg. Beih. 35: 21 (1925).

Habenaria arechavaletae var. obovatipetala (Schltr.) Pabst, Rodriguésia 16-17: 130 (1954).

Distribución: Brasil, Uruguay (CER, LAV, MAL, MON, RIV, SAN).

Ambientes: BS, BP, BC.

ECO-REGIONES: EsCr, SiEs, CuSeGo.

HÁBITO: Terrestre.

ESTADO DE CONSERVACIÓN: No consta en Marchesi et al. (2013).

Material examinado: URUGUAY. Cerro Largo, Fraile Muerto, I-1986, Izaguirre s.n. (MVFA 18065). Lavalleja, Cerro de Minas, 06-II-1952, Teague s.n. (MVM 15229).

Maldonado,Cerro de las Animas, 20-I-1937, Rosengurtt B2422 (MVFA). Montevideo, Carrasco, 1915, Berro 7829 (MVFA). Rivera, Aurora, 16-II-2001, Brussa \& Grela s.n. (MVFA 32748) San José, Barra de Santa Lucía, 16-I-1932, Osten 22453A (MVM).

NotA: Pabst (1952) y Batista et al. (2011b) indican su distribución en el país.

40. Habenaria paivaeana Rchb.f., Xenia Orchid. 3:

17 (1878).

Habenaria chloroceras Kraenzl., Repert. Spec. Nov. Regni Veg. 1: 89 (1905).

Distribución: Argentina, Bolivia, Perú, Uruguay (CER, MAL, MON, SAN).

Ambientes: BC, BR, $\mathrm{H}$.

ECo-REgiones: GrSaLu, GrLaMe.

НÁBito: Terrestre.

Estado de Conservación: Prioritaria (Criterio 3).

Material examinado: URUguay. Cerro Largo, Río Tacuarí, 10-XII-1965, Arrillaga et al. 2349 (MVFA).

Maldonado, San Carlos, 28-XII-1906, Berro 3628 (MVFA). Montevideo, Carrasco, 16-III-1913, Osten 6496 (MVM). San José, Barra de Santa Lucía, 09-I-1931, Osten 22172 (MVM).

NotA: Batista et al. (2011b) indica su distribución en el país.

41. Habenaria parviflora Lindl., Gen. Sp. Orchid. Pl.: 314 (1835). 
Habenaria uruguayensis Garay, Bradea 2: 118 (1976).

Habenaria montevidensis f. parviflora (Lindl.) Pabst, Orquídea (Rio de Janeiro) 19: 84 (1957).

Habenaria reichenbachiana Barb.Rodr., Gen. Sp. Orchid. 1: 159 (1877).

Habenaria montevidensis var. tucumanensis Griseb., Abh. Königl. Ges. Wiss. Göttingen 24: 340 (1879).

Habenaria angulosa Barb.Rodr., Gen. Spec. Orchid. 2: 258 (1882).

Habenaria caaguazuensis Cogn., Bull. Soc. Roy.

Bot. Belgique 43: 277 (1907).

Distribución: Argentina, Brasil, Colombia, Ecuador, Paraguay Uruguay (CAN, CER, COL, DUR, FLI, LAV, MAL, MON, PAY, RIO, RIV, ROC, SAL, SAN, SOR, TAC).

AmbIentes: BP, BS, BQ, BC, BR, H, P.

Eco-regiones: CuSeOe, $\mathrm{CuBa}, \mathrm{CuSeGo}, \mathrm{SiEs}, \mathrm{GrSaLu}$, GrLaMe, EsCr.

HÁBITO: Terrestre.

ESTADO DE CONSERVACIÓN: No consta en Marchesi et al. (2013).

Material examinado: URUguay. Canelones, Balneario Guazubirá Nuevo, 27-II- 2017, Lafargue s.n. (MVJB 30363). Cerro Largo, Arevalo, 13-III-2015 Muñoz s.n. (MVJB 29675). Colonia, Ruta 12, 1986, Izaguirre s.n. (MVFA32143). Durazno, Villa del Carmen, 2002, Izaguirre s.n. (MVFA 32856). Florida, Arroyo Molles, 09-XI-2016 Brussa et al. s.n. (MVJB 30534). Lavalleja, Minas, 12-I-2015, Muñoz s.n. (MVJB 29688). Maldonado, Cerro del Toro, 06-I-2019, Marin et al. OR10 (MVJB). Montevideo, Playa La Colorada, 17-XII-2001, Brussa s.n. (MVJB 23622). Paysandú, Ruta 26, 1977, Marchesi s.n. (MVFA 14281). Río Negro, Palleros, 1941, Gallinal et al. PE4620 (MVFA). Rivera, Cerro Chato Dorado, 2002, Izaguirre s.n. (MVFA32646). Rocha, Santa Teresa, 05-II-2019, Marín et al. OR37 (MVJB). Salto, Termas de Arapey, 25-XI-2001, Seijo et al. 2396 (SI). San José, Sierra de Mahoma, 1998, Izaguirre s.n. (MVFA 28057). Soriano, Mercedes, 1914, Berro 7545 (MVFA). Tacuarembó, Ciudad de Tacuarembó, 1957, Rosengurtt B6917 (MVFA).

Nota: Su distribución en Uruguay es citada en Pabst (1952), Batista et al. (2011b) y Sanguinetti (2015) que indica su sinonimia con $H$. uruguayensis.

42. Habenaria pentadactyla Lindl., Gen. Sp. Orchid. P1.: 307 (1835).

DistriBución: Argentina, Brasil, Uruguay (CAN,
MAL, MON, RIV, ROC, SAN).

Ambientes: BR, BP, BC, BQ, BS.

Eco-Regiones: GrSaLu, GrLaMe, CuSeGo, $\mathrm{CuBa}$. HÁBito: Terrestre.

Estado de Conservación: Prioritaria (Criterio 1, 4).

Material examinado: URUguaY. Canelones, Santa Ana, 07-II-2004, Haretche 5 (MVJB 21649). Maldonado, Piriápolis, 28-I-1911, Osten 5515 (MVM). Montevideo, Carrasco, 06-II-1935, Legrand 72 (MVM). Rivera, Bajada de Pena, 12-XII-1997, Marchesi \& Grela s.n. (MVFA 27110). Rocha, Laguna Negra, 20-III-1938, Rosengurtt B2611 (MVFA). San José, Barra de Santa Lucía, 28-I-1919, Osten 14720 (MVM).

Nota: Su distribución en Uruguay es citada en Pabst (1952) y Batista et al. (2011b).

43. Habenaria platanthera Rchb.f., Linnaea 41: 55 (1876).

Distribución: Brasil, Uruguay (MAL).

Ambiente: BC.

ECO-REGIÓN: GrLaMe.

HÁBito: Terrestre.

Estado DE CONSERVACIÓn: Posiblemente extinta en el país, última colecta conocida hace más de 100 años en un área actualmente urbanizada.

Material examinado: URUGUay. Maldonado, "in dunis", 1869, Gibert 888 (MVM).

Nota: Batista et al. (2011b) indican su distribución en el país.

44. Habenaria repens Nutt., Gen. N. Amer. Pl. 2: 190 (1818).

Habenaria aranifera Lindl., Gen. \& Spec. Orch. P1.: 313.1835 .

Mesicera repens (Nutt.) Raf., Fl. Tellur.: 2: 39 (1837).

Platanthera repens (Nutt.) Alph.Wood, Class-book Bot. 41: 685 (1861).

Orchis repens (L.) Eystr. ex Poir. Encycl. 6(2): 581 (1805), nom. inv.

Habenaria polygonoides Schltr., Feddes Repert. Spec. Nov. Regni Veg. 16:252 (1919).

Distribución: Argentina, Bolivia, Brasil, Colombia, Ecuador, Guayana Francesa, Paraguay, Perú, Surinam, Uruguay (CAN, MAL, MON, ROC, SAN), Venezuela.

Ambientes: BR, BC, $\mathrm{H}$.

Eco-Regiones: GaSaLu, GrLaMe, EsCr.

НÁвіто: Terrestre. 
Estado DE CONSERVACIÓN: No consta en Marchesi et al. (2013).

Material examinado: URUGUay. Canelones, La Floresta, 26-XII-1937, Lombardo 1780 (MVFA). Maldonado, en dunas, 17-I-1900, Osten 4037 (MVM). Montevideo, Carrasco, 1913, Osten 6494 (MVM). Rocha, La Paloma, 1993, Izaguirre s.n (MVFA 21657). San José, Barra de Santa Lucía, 15-I-1932, Osten 22450 (MVM).

NотA: Batista et al. (2011b) indican su distribución en el país.

Malaxis Sol. ex Sw.

45. Malaxis irmae Radins \& Salazar, Phytotaxa 175(3): 126 (2014).

DistRiBuCión: Argentina, Uruguay (LAV).

Ambiente: BS.

ECO-REGIÓN: SiEs.

HÁBito: Terrestre.

Estado DE CONSERVACiÓn: Prioritaria (Criterio 3).

Material examinado: URUGUAY. Florida, 20-IV-2009, Marchesi s.n. (MVFA 34842 no visto). Lavalleja, Cerro Gigante, 2017, Muñoz \& Diaz s.n. (MVJB 30501).

NotA: Izaguirre et al. (2010) y Radin et al. (2014) citan el espécimen en Florida, no fue localizado.

46. Malaxis parthoni C.Morren, Bull. Acad. Roy. Sci. Bruxelles 5: 485 (1839).

Microstylis parthonii (C.Morren) Rchb.f., Ann. Bot. Syst. 6: 206 (1861).

Malaxis disepala (Rchb.f.) Kuntze, Revis. Gen. P1. 2: 673 (1891).

Microstylis ovatilabia Schltr., Repert. Spec. Nov. Regni Veg. 16: 330 (1920).

Malaxis argentinensis L.O.Williams, Lilloa 4: 364 (1939).

DistRIBUCIÓN: Argentina, Uruguay (CER).

Ambiente: $\mathrm{H}$.

ECO-REGIÓN: sin información.

HÁBIto: Terrestre

Estado de Conservación: Prioritaria (Criterio 2, 3).

Material eXaminado: Cerro Largo, 18-VII-2008, Berazategui \& Duarte s.n. [(MVFA 34841) citada en Izaguirre et al. (2010), no visto; 22-VIII-2009, Berazategui et al. s.n. [(MVM 20269) citada en Izaguirre et al. (2010), no visto].

NotA: Izaguirre et al. (2010) citan estos espécimen como únicas colectas en el país sugiriendo las características poco vistosa de su floración y distribución como factores influyentes.

\section{Orthochilus Hochst. ex A.Rich.}

47. Orthochilus ruwenzoriensis (Rendle) Bytebier, Taxon 63(1): 20 (2014)

Eulophia ruwenzoriensis Rendle, J. Bot. 33: 166 (1895).

Pteroglossaspis ruwenzoriensis (Rendle) Rolfe, Fl. Trop. Afr. 7: 100 (1897).

Distribución: Argentina, Bolivia, Brasil, Paraguay, Uruguay (MAL, RIO).

AMBIENTE: BS.

ECO-REGIÓN: GrLaMe.

HÁBito: Terrestre.

Estado de Conservación: Prioritaria (Criterio 2, 3).

Material examinado: URUGUay. Maldonado, Parque Maldonado, 16-III-1921, Osten 15746 (MVM); idem, 14II-1923, Osten 15746 (MVM). Río Negro, en cultivo de Eucalyptus sp, 07-II-2002, Ramos \& Beasley s.n. (MVFA 32219).

Nota: Registrada a principios del sigo pasado como Pteroglossaspis argentina Rolfe y rencontrada hace algunos años en bosques cultivados de Eucalyptus sp. (Izaguirre 2010). Martos et al. (2014) estudiaron los clados de Eulophia y restablecieron el genero Orthochilus para uno de estos.

Pelexia Poit. ex Lindl.

48. Pelexia arechavaletae (Mytnik, Szlach. \& Górniak) J.M.H.Shaw, Orchid Rev. 122: 76 (2014).

Pachygenium arechavaletae Mytnik, Szlach. \& Górniak, Ann. Bot. Fenn. 47(3): 218 (2010).

Distribución: Uruguay (MON). Endémica del país.

AMBIEnTE: BR.

ECO-REGIÓN: GrSaLu

HÁBITO: Terrestre.

EstAdO DE CONSERVACIÓN: No consta en Marchesi et al. (2013). Cumple con los criterios 1, 2, 3, se sugiere adicionar a la lista de especies prioritarias para el país. Material examinado: URUGUAY. Montevideo, Barra de Santa Lucía, s.f., Arechavaleta s.n. [(P) Citado en MytnikEjsmont et al. (2010), no visto].

49. Pelexia bonariensis (Lindl.) Schltr., Beih. Bot. Centralbl., Abt. 2, 37(3): 400 (1920).

Spiranthes bonariensis Lindl., Gen. Sp. Orchid. P1.: 475 (1840). 
Gyrostachys bonariensis (Lindl.) Kuntze, Revis. Gen. Pl. 1: 664 (1891).

Stenorrhynchos bonariense (Lindl.) Cogn., Fl. Bras. 3(4): 164 (1895).

Pachygenium bonariense (Lindl.) Szlach., R.González \& Rutk., Polish Bot. J. 46(1): 4 (2001).

Pachygenium saltense (Griseb.) Szlach., R.González \& Rutk., Polish Bot. J. 46(1): 6 (2001). Pachygenium callosum Mytnik, Szlach. \& Górniak, Ann. Bot. Fenn. 47: 215 (2010).

Distribución: Argentina, Bolivia, Paraguay, Perú, Uruguay (ROC, TAC).

Ambientes: BS, P.

ECo-REgiones: GrLaMe, CuBa.

HÁBito: Terrestre.

ESTADO DE CONSERVACIÓN: No consta en Marchesi et al. (2013).

Material examinado: URUGUAY. Rocha, Fortaleza de Santa Teresa, 20-II-1938, Rosengurtt B2628 (MVM). Tacuarembó, Cerro de Tacuarembó, IV-1924, Osten 17119 (MVM).

50. Pelexia lindmanii Kraenzl., Kongl. Svenska Vetensk. Acad. Handl., n.s., 46(10): 18 (1920).

Pelexia stictophylla Schltr., Repert. Spec. Nov.

Regni Veg. Beih. 35: 36 (1925).

Distribución: Argentina, Brasil, Paraguay, Uruguay (RIO, ROC).

AMBIENTE: BR, $\mathrm{H}$.

ECO-REGIÓN: GrLaMe.

HÁBito: Terrestre.

Estado de CONSERVACIÓN: Prioritaria (Criterio 3).

Material eXaminado: URUGUAY. Rocha, Santa Teresa, I-2003, Beyhaut s.n. (MVFA 32839).

Prescottia Lindl.

51. Prescottia oligantha (Sw.) Lindl., Gen. Sp. Orchid. Pl.: 454 (1840).

Cranichis oligantha Sw., Prodr. Veg. Ind. Occ.: 120 (1788).

Cranichis micrantha Spreng., Syst. Veg. 3: 700 (1826), nom. Superfl.

Prescottia micrantha Lindl., Edwards's Bot. Reg. 22:1915 (1836).

Prescottia tenuis Lindl., Gen. Sp. Orchid. Pl.: 454 (1840).

Prescottia myosurus Rchb.f. ex Griseb., Fl. Brit. W. I. 640 (1864).
Prescottia microrhiza Barb.Rodr., Gen. Sp. Orchid. 1: 179, (1877).

Prescottia pubescens Barb.Rodr., Gen. Sp. Orchid. 1: 178 (1877).

Prescottia nivalis Barb.Rodr., Gen. Sp. Orchid. 2: 278 (1881).

Distribución: Argentina, Bolivia, Brasil, Colombia, Ecuador, Perú, Paraguay, Uruguay (CAN).

AMBIENTE: BC.

ECO-REGIÓN: GrSaLu.

HÁBITo: Terrestre.

ESTADO DE CONSERVACIÓN: No consta en Marchesi et al. (2013).

Material examinado: URUguay. Canelones, La Floresta, 1923, Osten 16918 (MVM).

52. Prescottia ostenii Pabst, Bradea 3(3): 19 (1979).

DistriBución: Brasil, Uruguay (CAN).

AmBiente: BC.

ECO-REGIÓN: GrSaLu.

НÁвіто: Terrestre.

Estado de Conservación: Prioritaria (Criterios 2, 3), Alonso-Paz \& Bassagda (2006) indican que probablemente se encuentre extinta en el país.

Material examinado: URUGUAY: Canelones, La Floresta, 1933, Osten 22939 (MVM); La Floresta 30-IX1923, Osten 16928 (MVM).

\section{SAColla Raf.}

53. Sacoila lanceolata (Aubl.) Garay, Bot. Mus. Leafl. 28(4): 352, t. 45 (1982).

Limodorum lanceolatum Aubl., Hist. Pl. Guiane 2: 821 (1775).

Neottia lanceolata (Aubl.) Willd., Sp. Pl., 4(1): 73 (1805).

Stenorrhynchos lanceolatum (Aubl.) Rich., De Orchid. Eur.: 37 (1817).

Gyrostachys lanceolata (Aubl.) Kuntze, Revis. Gen. P1. 2: 664 (1891).

Spiranthes lanceolata (Aubl.) León, Contr. Ocas. Mus. Hist. Nat. Colegio "De La Salle" 8: 358 (1946).

Sacoila paludicola (Luer) P.M.Br., N. Amer. Native Orchid J. 14: 187 (2008).

Distribución: Argentina, Bolivia, Brasil, Colombia, Ecuador, Guayana Francesa, Perú, Paraguay, Surinam, Uruguay (RIO, RIV, TAC), Venezuela.

LANKESTERIANA 20(3). 2020. (C) Universidad de Costa Rica, 2020. 
Ambientes: BS, BQ, P.

ECo-Regiones: CuSeGo, $\mathrm{CuBa}$.

HÁBIto: Terrestre.

Estado de CONSERVACión: No consta en Marchesi et al. (2013).

Material Examinado: URUGUAY. Río Negro, Estancia El Rosario, 19-XI-1998, Marchesi \& Vignale s.n. (MVFA 28581). Rivera, Ruta 30, 228 km, 22-X-2005, Delfino s.n. (MVJB 23508); Ruta $27 \mathrm{Km} \mathrm{8,} \mathrm{15-X-2003,} \mathrm{Grela} \mathrm{\&}$ Brussa 1113 (MVJB). Tacuarembó, Establecimiento El Cerro, 22-X-2005 Brussa s.n (MVJB 23752); Ruta 5, 15XI-1996, Grela \& Romero s.n. (MVFA 26290); Ciudad de Tacuarembó, 25-X-1991, Lombardo V. s.n. (MVFA 20302); Gruta de los Cuervos, 08-XI-1987, Denis \& Scarlato s.n. (MVJB 21304)

\section{Skeptrostachys Garay}

54. Skeptrostachys arechavaletanii (Barb.Rodr.) Garay, Bot. Mus. Leafl. 28(4): 359 (1980 publ. 1982). Stenorrhynchos arechavaletanii Barb.Rodr., Contr. Jard. Bot. Rio de Janeiro 4: 99 (1907).

Stenorrhynchos exaltatum Kraenzl., Kongl. Svenska Vetensk. Acad. Handl., n.s., 46(10): 29 (1911). Stenorrhynchos lateritium Kraenzl., Kongl. Svenska Vetensk. Acad. Handl., n.s., 46(10): 21 (1911).

DisTRIBUCIÓN: Brasil, Uruguay (CER, LAV, MAL, RIV, SAN, TAC)

Ambientes: BS, BC, P, BQ.

Eco-regiones: GrLaMe, $\mathrm{CuBa}, \mathrm{CuSeGo,} \mathrm{EsCr.}$

HÁBIto: Terrestre.

ESTADO DE CONSERVACIÓN: No consta en Marchesi et al. (2013).

Material eXaminado: URUGUAY. Cerro Largo, Ruta 8 $377,5 \mathrm{~km}, 12-\mathrm{XII}-1986$, Armand et al. s.n. (MVFA 18919). Lavalleja, Estancia Los Ceibos, 03-I-2018, Brussa \& Brussa s.n. (MVJB 31038). Maldonado, Punta Ballena, 21 I-2019, Marin \& Marin OR17 (MVJB). Rivera, Camino de Curticeira a paso Ataques. COFUSA, 15-II-2001, Brussa \& Grela s.n. (MVFA 32750). San José, Sierra de Mahoma, 29IV-1993, Marchesi et al s.n. (MVFA 21962). Tacuarembó, Sierra del Infiernillo, 04-II-1994, Bayce et al. s.n. (MVFA 23931).

55. Skeptrostachys balanophorostachya (Rchb.f. \& Warm.) Garay, Bot. Mus. Leafl. 28(4): 359 (1982).

Spiranthes balanophorostachya Rchb.f. \& Warm., Otia Bot. Hamburg.: 2: 84 (1881).

Skeptrostachys montevidensis (Barb. Rodr.) Garay,
Bot. Mus. Leafl. 28(4): 360 (1982).

Stenorrhynchos balanophorostachyum (Rchb.f. \& Warm.) Cogn. Bull. Herb. Boissier, sér. 2, 3(10): 931 (1903) nom. Inv.

Stenorrhynchos polyanthum Kraenzl., Kongl. Svenska Vetensk. Acad. Handl., n.s., 46(10): 30 (1911).

Skeptrostachys stenophylla (Cogn.) Szlach., Fragm. Florist. Geobot. 39(2): 418 (1994).

Stenorrhynchos montevidense Barb.Rodr., Contr. Jard. Bot. Rio de Janeiro 4: 98 (1907).

Stenorrhynchos stenophyllum Cogn., Bull. Soc. Roy. Bot. Belgique 43: 289 (1907).

DistribuCiÓn: Brasil, Paraguay, Uruguay (CER, DUR, MAL, PAY, RIV, ROC, SAL, SAN).

AMBIENTES: BS, P, BR, BPa.

Eco-regiones: GrSaLu, GrLaMe, CuBa, CuSeGo, SiEs.

HÁBIto: Terrestre.

ESTADO DE CONSERVACIÓN: No consta en Marchesi et al. (2013).

Material examinado: URUguay. Cerro Largo, Arevalo, 13-III-2015, Muñoz \& Díaz s.n. (MVJB 29677). Durazno, Arroyo Sarandí del Yi, 23-III-1971, Calero et al. s.n. (MVFA 16826). Maldonado, Pan de Azúcar, 09IV-2016, Brussa \& Muñoz s.n. (MVJB 30113). Paysandú, en vía férrea, 12-III-1971, Del Puerto \& Marchesi s.n. (MVFA 10401). Rivera, Ruta 5, 438 km, 27-III-1985, Marchesi et al. s.n. (MVFA 17548). Rocha, Estancia El Palmar, 20-III-1977, Marchesi s.n. (MVFA 14027). Salto, Ruta 3, 484 km, 19-II-1994, Izaguirre et al. s.n. (MVFA 20087). San José, Barra de Santa Lucía, IV-1996, Lombardo 633 (MVFA).

56. Skeptrostachys berroana (Kraenzl.) Garay, Bot. Mus. Leafl. 28(4): 359 (1982).

Stenorrhynchos berroanum Kraenzl., Kongl. Svenska Vetensk. Acad. Handl., n.s., 46(10): 26 (1911).

Pelexia berroana (Kraenzl.) Schltr., Beih. Bot. Centralbl., 37(3): 400 (1920).

DistribuCiÓn: Uruguay (LAV, MAL, ROC). Endémica del país.

Ambientes: BS, P, BC.

ECO-REGIONES: SiEs, GrLaMe.

HÁBito: Terrestre.

Estado de COnSERvación: No consta en Marchesi et al. (2013). Cumple con los criterio 1, 2, 3, se sugiere adicionar a la lista de especies prioritarias para el país. 
Material EXAMINADO: URUGUAY. Lavalleja, Minas, Cerro del Verdún, 03-II-1911, Berro 4306 (MVFA). Maldonado, Piriápolis, II-1907, Arechavaleta 32 (MVFA). Rocha, La Coronilla, 27-II-1972, Izaguirre s.n. (MVFA 2796).

57. Skeptrostachys gigantea (Cogn.) Garay, Bot. Mus. Leafl. 28(4): 359 (1982).

Stenorrhynchos giganteum Cogn., Fl. Bras. 3(6): 533 (1906).

Distribución: Argentina, Brasil, Paraguay, Uruguay (LAV, MAL, TAC, TRE).

AmbIentes: BS, BC, BQ, P.

ECo-Regiones: SiEs, GrLaMe, CuBa, CuSeGo.

HÁBito: Terrestre.

ESTADO DE CONSERVACIÓN: No consta en Marchesi et al. (2013).

Material examinado: URUGUAY. Lavalleja, Establecimiento Papazian, 10-II-2017, Muñoz \& Díaz s.n. (MVJB 30602); Cascada del Penitente, 19-III-1943, Legrand 4947 (MVFA). Maldonado, Punta Ballena, 21-I-2019, Marin OR15 (MVJB); Punta Ballena, 29-I1971, Izaguirre 2787 (MVFA); Punta Ballena, 04-IV1903, Arechavaleta s.n. (MVFA); Pan de Azúcar, s.f. Arechavaleta s.n. (MVFA); Piriapolis, Cerro Inglés, 7-II1910, Jappen s.n. (MVM 5315). Rocha, San Miguel, 10-II1985, Marchesi s.n. (MVFA 8344). Tacuarembó, Cuchilla de la Casa de Piedra, 25-I-1977, Correa 11631 (SI). Treinta y Tres, Estancia La Teja, 20-I-2010, Haretche et al. 222 (MVJB).

58. Skeptrostachys paraguayensis (Rchb.f.) Garay, Bot. Mus. Leafl. 28(4): 360 (1982).

Spiranthes paraguayensis Rchb.f., Linnaea 25: 230 (1852).

Stenorrhynchos paraguayense (Rchb.f.) Cogn. Bull. Herb. Boissier, sér. 2, 3: 931 (1903)

Stenorrhynchospachystachyum Kraenzl., Kongl.

Svenska Vetensk. Acad. Handl., n.s., 46(10): 30 (1911).

Skeptrostachys stenorrhynchoides Szlach., Fragm.

Florist. Geobot. 39(4): 418 (1994).

DistriBuCión: Argentina, Brasil, Paraguay, Uruguay (CAN, PAY, RIO, ROC).

AMBIENTES: BS, BP, H, P.

ECO-REgiones: GrLaMe, GrSaLu, $\mathrm{CuBa}, \mathrm{CuSeO}$.

HÁBITO: Terrestre.

Estado de CONSERVACIÓn: Prioritaria (Criterio 3).

Material examinado: URUguay. Canelones, Santa
Lucía, 1869, Gibert 893 (K). Lavalleja, Sierra de Minas, s.f., Arechavaleta s.n. (MVFQ 176). Paysandú, Meseta Artigas, 7-V-1964, Del Puerto \& Marchesi 3557 (MVFA); Meseta Artigas, 03-V-1969, Del Puerto \& Marchesi 3557 (MVFA). Río Negro, Campo El Jabalí, 02-IV-1998, Marchesi \& Vignale s.n. (MVFA 28153); Campo Echevarne, 09-V1996, Marchesi \& Vignale s.n. (MVFA 25498); Campo Echevarne, 20-V-1996, Marchesi \& Vignale s.n. (MVFA 25749); Campo El Jabalí, 11-IV-1994, Marchesi \& Vignale s.n. (MVFA 28154). Rocha, Sierra de Rocha, 17-VI-1999, Báez s.n. (MVFA 32157); Parque San Miguel, 24-III-1966, Del Puerto \& Marchesi 6134 (MVFA); La Coronilla, 27-II1972, Izaguirre 2795 (MVFA).

Trichocentrum Poepp. \& Endl.

59. Trichocentrum pumilum (Lind1.) M.W.Chase \& N.H.Williams, Lindleyana 16(2): 138 (2001).

Oncidium pumilum Lindl., Bot. Reg. 11: t. 920 (1825).

Lophiaris pumila (Lindl.) Braem, Schlechteriana 4(1-2): 21 (1993).

Epidendrum ligulatum Vell., Fl. Flumin. 9: t. 15 (1831), nom. Illeg.

Oncidium pumilum var. angustifolium Cogn., Fl. Bras. 3(6): 374 (1905).

Oncidium pumilum var. robustum Cogn., Fl. Bras. 3(6): 374 (1905).

Oncidium pumilum var. laxum Kraenzl., Ark. Bot. 16(8): 27 (1921).

Oncidium minutiflorum Schltr., Repert. Spec. Nov. Regni Veg. 21: 341 (1925).

DistribuCIÓn: Argentina, Brasil, Paraguay, Uruguay (CER, ROC, TRE).

AMBIENTES: BR, BQ.

ECO-REGIÓN: GrLaMe.

HÁBITo: Epífita.

Estado de Conservación: Prioritaria (Criterio 3, 4).

Material examinado: URUGUAY. Cerro Largo, Establecimiento Sofía, 15-XII-2014, Brussa s.n. (MVJB 29205). Rocha, Balneario Saglia, Laguna Merin, 21-X2012, Brussa \& Gago s.n. (MVM 28374); Río Cebollatí, 28-XI-1989, Marchesi et al. s.n. (MVFA 19427). Treinta y Tres, Río Tacuarí, 11-XII-2014, Rossado 411 (MVM); Río Cebollatí, 17-XII-2002, Izaguirre s.n. (MVFA 32860); Río Cebollatí, 18-I-1937, Legrand 1047 (MVM); Río Cebollatí, 30-XII-1905, Berro 2858 (MVM).

NotA: Indicada para el país en Mai et al. (2019) e Izaguirre (2013). 


\section{TAXONES NO INCLUÍDOS}

Brachystele delicatula (Kraenzl.) Schltr., Beih. Bot. Centralbl. 37(3): 373 (1920).

Spiranthes delicatula Kraenzl., Bot. Jahrb. Syst. 36(80): 9 (1905).

Material eXAminado: No visto

Nota: Citada en Schinini et al. (2010), existe un posible error en la localidad por lo que no se localizaron los registros para Uruguay. Además, el typus pertenece a Paraguay.

Cyclopogon bicolor (Ker Gawl.) Schltr., Repert. Spec. Nov. Regni Veg. Beih. 6: 52 (1919).

Neotia bicolor Kerl-Gawl, Bot. Reg. 10 t. 794.1824.

Gyrostachys bicolor(Ker Gawl.) Kuntze, Rev. Gen. 2: 664.1891.

Beadlea bicolor (Kerl-Gawl.) Garay, Bot. Mus. Leafl. 28 (4): 299.1980.

Spiranthes bicolor (Kerl-Gawl) Lindl., Bot. Reg. 10: subt. t. 823:(1824).

Cyclopogon bicolor (Lindl.) Schltr. var. minor Hoehne, Fl. Bras. 1945.

NотA: Su distribución en Uruguay es citada en Schinini (2010), Andrade et al. (2019) y en La Flora del Cono Sur (2020), sin embargo los especímenes identificado como C. bicolor [Florida, Arroyo Mansavillagra, Rosengurtt, Gallinal 5763 (MVM). Cerro Colorado, 15-X-1943, Rosengurtt 5333 (MVM); Lavalleja, Minas, 2-XI-1921, Osten 16050 (MVM); San José, Sierra de Mahoma, 27-X-1940, Rosengurtt B3158 (MVM)] corresponden a C. elatus.

Cyclopogon dutraei Schltr., Repert. Spec. Nov. Regni Veg. Beih. 35: 30 (1925).

Beadlea dutraei (Schltr.) Garay, Bot. Mus. Leafl. 28(4): 300 (1982).

Nota: Su distribución en Uruguay es citada por Andrade et al. (2019) y La Flora del Cono Sur (2020) con base en Pabst (1952a); sin embargo, los especímenes citados para el país corresponden a C. elatus.

Gomesa flexuosa (G.Lodd.) M.W.Chase \& N.H.Williams, Phytotaxa 1: 58 (2009).

Oncidium flexuosum G.Lodd., Bot. Cab. 5: t. 424 (1820).

Nota: Taxón con especímenes cultivados [Canelones, cultivada, origen Cuchilla Verde, 12-XII-2002, Izaguirre s.n (MVFA 32855). Colonia, cultivada en Montevideo, origen Colonia, 20-XII-1966, Arrillaga 2542 (MVFA)].

Habenaria imbricata Lindl., Gen. Sp. Orchid. PI. 313(1835).

Habenaria graciliscapa Barb.Rodr., Gen. Sp. Orchid. 1: 155(1877).

NotA: Andrade et al. (2019) indica la ocurrencia para el país. Batista et al. (2011a) coloca la distribución de esta especie unicamente para Brasil e indica que los especímenes de Pabst (1952) para Uruguay no fueron confirmados. El espécimen examinado y citado por Pabst (1952) como H.graciliscapa [Canelones. Bañados de los Arroyos Negro y Pando. 11-II-1947. Legrand 1956 (MVM)] se trata de H. parviflora (identificada como H. uruguayensis).

Habenaria inconspicua Cogn., Bull. Soc. Roy. Bot. Belgique 43: 274 (1907).

Material examinado: URUGUay. Canelones, 1931, Herter 86651 (MVM No visto).

Nota: Citada en Pabst \& Dungs (1975), Andrade et al. (2019) y en La Flora del Cono Sur (2020). Batista et al. (2011a) la consideran sinónimo de H. edwalli y con distribución unicamente en Brasil. El material examinado no fue localizado.

Agradecimientos. A los curadores de los herbarios MVM, SI, MVFA y MVJB Manuel García, Manuel Belgrano, Mauricio Bonifacino y Federico Aretche por la gran colaboración y atención prestada en el acceso a las colecciones. A Fabián Muñoz director del Jardín Botánico Atilio Lombardo de Montevideo y el investigador Andrés González por la amplia información compartida y contribuir con gran cantidad de fotos de excelente calidad. A todas las personas que contribuyeron en la colecta de Orchidaceae de Uruguay, especialmente a Marina Diaz, Yamandú Marín y Darío Frost. 


\section{LITERATURA CITADA}

Achkar, M., Brazeiro, A. \& Bartesaghi, L. (2012). Principales amenazas para la conservación de la biodiversidad de Uruguay. En: A. Brazeiro (Ed.), Eco-regiones de Uruguay: Biodiversidad, presiones y conservación. Aportes a la Estrategia Nacional de Biodiversidad. (70-85). Montevideo, Uruguay: Facultad de Ciencias/Vida Silvestre/ SZU/CIEDUR.

Alonso-Paz, E. \& Bassagda, M., J. (2006). Flora y vegetación de la costa platense y atlántica uruguaya. En: R Menafra, L. Rodríguez-Gallego, F. Scarabino \& D. Conde (Eds.), Bases para la conservación y el manejo de la costa uruguaya. (71-88). Montevideo, Uruguay: Vida Silvestre Uruguay.

Andrade, B. O., Marchesi, E., Burkart, S., Setubal, R. B., Lezama, F., Perelman, S., Schneider, A. A., Trevisan, R., Overbeck, G. E. \& Boldrini, I. I. (2018). Vascular plant species richness and distribution in the Río de la Plata grasslands. Botanical Journal of the Linnean Society, 188(3), 250-256.

Anilkumar, A.(2004). Vanilla cultivation:A profitable agribased enterprise. Kerala Calling, 2, 26-30.

APG IV. (2016). An update of the Angiosperm Phylogeny Group classification for the orders and families of flowering plants: APG IV. Botanical Journal of the Linnean Society,181, 1-20.

Arechavaleta, J. (1894). Flora uruguaya. Uruguay: Anales del Museo Nacional de Montevideo.

Batista, J., Bianchetti, L., González-Tamayo, R., Figueroa, X. \& Cribb, P. J. (2011a). A synopsis of new world Habenaria (Orchidaceae) I. Harvard Papers in Botany, 16(1), 1-47.

Batista, J., Bianchetti, L., González-Tamayo, R., Figueroa, X. \& Cribb, P. J. (2011b). A synopsis of new world Habenaria (Orchidaceae) II. Harvard Papers in Botany, 16(2), 233-273.

Blum, A. (Ed). (2015). Atlas de Cobertura del Suelo del Uruguay. Cobertura del Suelo y Detección de Cambios $2000-2011$. Montevideo, Uruguay: Food and Agriculture Organization of the United Nations (FAO/DINOT).

Brazeiro,A.,Panario,D., Soutullo,A., Gutierrez,O., Segura,A.\&MaiP.(2015a).Identificaciónydelimitación deeco-regionesde Uruguay. En:A. Brazeiro(Ed.),Eco-regiones de Uruguay: Biodiversidad, presionesyconservación. Aportes a la Estrategia Nacional de Biodiversidad (46-59). Montevideo, Uruguay: Facultad de Ciencias (FCIEN/Vida Silvestre/SZU/CIEDUR).

Brazeiro, A., Soutullo, A. \& Bartesaghi, L. (2015b). Identificación de prioridades de conservación dentro de las eco-regiones de Uruguay. En: A. Brazeiro (Ed.), Eco-regiones de Uruguay: Biodiversidad, presiones y conservación. Aportes a la Estrategia Nacional de Biodiversidad. (60-69). Montevideo, Uruguay: Facultad de Ciencias (FCIEN/Vida Silvestre/ SZU/CIEDUR).

Brazeiro, A., Achkar, M., Canavero, A., Fagundez, C., Gonzalez, E., Grela, I., Lezama, F., Maneyro, R., Barthesagy, L., Camargo, A., Carreira, S., Costa, B., Nunez, D., da Rosa, I. \& Toranza, C. (2008). Prioridades geográficas para la conservación de la Biodiversidad terrestre de Uruguay. Resumen Ejecutivo. Montevideo, Uruguay: Facultad de Ciencias (UdelaR/FCIEN/PDT).

Brussa, C. (1996). Ecosistemas forestales nativos en Uruguay. Estado actual y grado de conservación. Montevideo, Uruguay: ESUCOBO - UICN Sur.

Brussa, C. \& Grela, I. (2007). Flora Arbórea del Uruguay. Con énfasis en las especies de Rivera y Tacuarembó. Rivera, Uruguay: Compañía Forestal Uruguay S.A.

Buzatto, C. R., Sanguinetti, A., Romero-González, G. A., van den Berg, C. \& Singer, R. B. (2014). A taxonomic synopsis of Brazilian Chloraeinae (Orchidaceae: Orchidoideae). Phytotaxa, 158(1), 1-22.

Buzatto, C. R., Singer, R. B., van den Berg, C., de Souza, É. R., \& Mota, B. M. (2020). Capanemia (Oncidiinae): an orchid genus revised and simplified. Plant Systematics and Evolution, 306(2), 1-19.

Buzatto, C. R., Singer, R. B., Romero-González, G. A., van den Berg, C. \& Salazar, G. A. (2013). Typifications and taxonomic notes in species of Brazilian Goodyerinae and Spiranthinae (Orchidaceae) described by José Vellozo and Barbosa Rodrigues. Taxon, 62, 609-621.

Cabrera, A. L. \& Willink, A. (1973). Biogeografía de América Latina. Washington D.C., EE.UU: Programa Regional de Desarrollo Científico y Tecnológico.

Cameron, K. M. (2011). Vanilla Orchids: Natural History and cultivation. Portland: Timber Press.

Castro, J. B. \& Singer, R. B. (2018). Nomenclature and taxonomy of Brazilian Gomesa species (Orchidaceae: Oncidiinae) described by João Barbosa Rodrigues. Taxon, 67(6), 1187-1193.

Chebataroff, J. (1960). Tierra uruguaya: introducción a la geografía física, biológica y humana del Uruguay . Montevideo, Uruguay: Don Bosco.

Chemisquy, A. (2013). Evaluación del estado de conservación de las especies del genero Gavilea (Orchidaceae, Chloraeinae) en Argentina y Chile. Revista del Museo Argentino de Ciencias Naturales nueva serie,15(2), 161-167.

Christenhusz, M. J., \& Byng, J. W. (2016). The number of known plants species in the world and its annual increase. Phytotaxa, 261(3), 201-217.

Correa, M. N. (1953). Un nuevo género y cuatro especies nuevas de orquídeas argentinas. Darwiniana,10, $157-168$.

Correa, M. N. (1955). Las orquídeas argentinas de la Tribu Polychondreae Schlechter: Subtribu Spiranthinae Pfitzer. Darwiniana,11(1), 24-88.

Correa, M. N. (1969). Chloraea, género sudamericano de Orchidaceae. Darwiniana, 15 (3), 374-415.

Cribb, P. (1999). Orchidaceae In: A. M. Pridgeon, P. J. Cribb, M. W. Chase \& F. N. Rasmussen (Eds.), Genera Orchidacearum vol. 1 (pp. 91-93). New York: Oxford University Press.

de Azevedo, C. O., van Den Berg, C. \& de Barros, F. (2014). A revision of Prescottia (Orchidaceae: Orchidoideae, Cranichideae). Phytotaxa, 178(4), 233-286. 
de Candolle, A. (1855). Geographie botanique raisonne?. Paris: Librairie de Masson.

del Puerto, O. (1987). Vegetación del Uruguay. Montevideo, Uruguay: Universidad de la República.

Dixon, A. P., Faber-Langendoen, D., Josse, C., Morrison, J. \& Loucks, C. J. (2014). Distribution mapping of world grassland types. Journal of Biogeography, 41(11), 2003-2019.

Dostálek, J. \& Frantík, T. (2008). Dry grassland plant diversity conservation using low-intensity sheep and goat grazing management: case study in Prague (Czech Republic). Biodiversity and conservation, 17(6), 1439-1454.

Dressler, R. L. (1993). Phylogeny and classification of the orchid family. Cambridge: Cambridge University Press.

Dressler, R. L. (2005). How many orchid species? Selbyana, 26, 155-158.

Evia, G. \& Gudynas, E. (2000). Ecología del paisaje del Uruguay. Aportes para la conservación de la diversidad biológica. Montevideo, Uruguay: Junta de Andalucía/MVOTMA.

Ferreira, P. M. \& Boldrini, I. I. (2011). Potential reflection of distinct ecological units in plant endemism categories. Conservation Biology, 25(4), 672-679.

Garay, L. (1982). Generic revision of the Spiranthinae. Cambridge: Botanical Museum Leaflets (Havard University).

García, S., Pezzani, F., Lezama, F. \& Paruelo, J. (2019). Los componentes del pastoreo afectan de forma diferencial las micorrizas en Paspalum dilatatum Poir. Ecología Austral, 29(2), 164-173.

Gibert, E. (1873). Enumeratio plantarum in agro montevidensi. Uruguay: Asociación Rural del Uruguay.

Gonçalves, F. (2016). Caracterização de populações de orquídeas em zonas sujeitas a pastoreio (Tesis Doctoral). Departamento de Ciências da Vida, Faculdade de Ciências e Tecnologia, Universidade de Coimbra, Lisboa.

Govaerts, R., Dransfield, J., Zona, S., Hodel, D. R. \& Henderson, A. (2020). World Checklist of Orchidaceae. Facilitated by the Royal Botanic Gardens, Kew. Recuperado de: http://wcsp.science.kew.org/ (Acceso Julio de 2020).

Grela, I. (2004). Geografía florística de las especies arbóreas de Uruguay: propuesta para la delimitación de dendrofloras.(Tesis de Maestría), PEDECIBA/Universidad de la República, Montevideo, Uruguay.

Haretche, F., Mai, P. \& Brazeiro, A. (2012). Woody flora of Uruguay: inventory and implication within the Pampean region. Acta Botanica Brasilica, 26(3), 537-552.

Herter, G. (1930). Florula Uruguayensis. Estudios botánicos de la región uruguaya IV. Plantae Vasculares. Orchidaceae. Montevideo, Uruguay: Imprenta Nacional.

Hurrel, J., Delucchi G. \& Correa M. (2009). Parte 3: Monocotiledóneas. En: Flora rioplatense: sistemática, ecología y etnobotánica de las plantas vasculares rioplatenses. Buenos Aires, Argentina: Literature of Latin Americ (LOLA).

Instituto Geográfico Militar (IGM). (2020). Información Territorial. Recuperado de: http://www.igm.gub.uy/ (Acceso Julio de 2020)

Instituto Uruguayo de Meteorología (INUMET). (2019). Clima. Recuperado de: http://www.inumet.gub.uy (Acceso junio de 2019).

IPNI. (2020). The International Plant Names Index.Recuperado de: http://www.ipni.org. (Acceso Junio de 2020).

Izaguirre, P. (1972). El genero Capanemia (Orchidaceae) en el Uruguay. Boletín de la Sociedad Argentina de Botánica, 14(3), $225-231$.

Izaguirre, P. (1973). Las especies uruguayas de Bipinnula (Orchidaceae). Boletín de la Sociedad Argentina de Botánica, $15(2-3), 261-276$.

Izaguirre, P. (1985). Las orquídeas silvestres del Uruguay. In: F. A. Baum (Ed.), Almanaque del Banco de Seguros del Estado 1985 (159- 164), Montevideo, Uruguay: Banco de Seguros del Estado.

Izaguirre, P. (2010). Novedades en orquídeas para Uruguay: primera contribución. Agrociencia Uruguay, 14, 1-9.

Izaguirre, P. (2013). Novedades en orquídeas para Uruguay: segunda contribución. Plantas Epífitas. Agrociencia Uruguay, $17,22-35$.

La Flora del Cono Sur. (2020). Instituto de Botánica Darwinion. Catalogo de Plantas Vasculares. Recuperado de darwin. edu.ar (Acceso Julio de 2020).

Lombardo, A. (1982). Flora montevidensis. Uruguay: Intendencia Municipal de Montevideo.

Lombardo, A. (1984). Flora montevidensis, Monocotiledoneas tomo III. Uruguay: Intendencia Municipal de Montevideo.

Mai, P. (2014). Flora epífita vascular de Uruguay con énfasis en helechos (Polypodiofitas). Tesis de Maestría. PEDECIBA/ UdelaR, Montevideo, Uruguay.

Mai, P., Rossado, A., Bonifacino, J. M. \& Waechter, J. L. (2019). Catalogue of the vascular epiphytic flora of Uruguay. Acta Botanica Brasilica, 33(4), 683-708.

Marchesi, E., Alonso, E., Delfino, L., García, M., Haretche. F. \& Brussa C. (2013). Plantas vasculares. In: A. Soutullo, C. Clavijo \& J. A. Martinez-Lanfranco (Eds.), Especies prioritarias para la conservación en Uruguay. Vertebrados, moluscos continentales y plantas vasculares. ( 27-72). Montevideo: Sistema Nacional de Áreas Protegidas (Snap/ Dinama/Mvotma y Dicyt/MEC).

Martos, F., Johnson, S. D., Peter, C. I., \& Bytebier, B. (2014). A molecular phylogeny reveals paraphyly of the large genus Eulophia (Orchidaceae): a case for the reinstatement of Orthochilus. Taxon, 63(1), 9-23.

MGAP. Ministerio de Ganadería, Agricultura y Pesca de Uruguay (Secretaria DIEA). (2015). Regiones Agropecuarias del Uruguay. Uruguay: Instituto Nacional de Investigación Agropecuaria.

MGAP. Ministerio de Ganadería, Agricultura y Pesca de Uruguay (2018). Estrategia Nacional de Bosque Nativo. Uruguay: Instituto Nacional de Investigación Agropecuaria.

Morrone, J. (2001). Biogeografía de América Latina y el Caribe. España: Programa Iberoamericano de Ciencia y Tecnología para el Desarrollo (CYTED). 
MVOTMA-Geoservicios. (2020a). Departamentos. Recuperado en:https://www.dinama.gub.uy (Acceso Julio de 2020).

MVOTMA-Geoservicios. (2020b). Cursos de agua. Recuperado en:https:/www.dinama.gub.uy/geoserver/u19600217/ (Acceso Diciembre de 2020).

Mytnik-Ejsmont, J., Szlachetko, D. L. \& Górniak, M. (2010). Taxonomical notes on Pachygenium (Orchidaceae). Annales Botanici Fennici, 47(3), 215-220.

Orden, E. A., Quiroga, A., Justiniano, D. R. \& Morlans, M. C. (2006). Efecto del sobrepastoreo en un pastizal de altura. Cumbres de Humaya. Catamarca. Argentina. Revista Ecosistemas, 15(3), 142-147.

Pabst, G. 1952. Orchidaceae Uruguayenses Collectorum Variorum Collectae. Rodriguesia, 15(27), 109-127.

Pabst, G. \& Dungs, F. (1975). Orchidaceae Brasilienses, vol. 1. Hildesheim: Brücke-Verlag Kurt Schmersow.

Pabst, G. \& Dungs, F. (1977). Orchidaceae Brasilienses, vol. 2. Hildesheim: Brücke-Verlag Kurt Schmersow.

Panario, D., Gutierrez, O., Achkar, M., Bartesaghi, L. \& Ceroni, M. (2015). Clasificación y mapeo de ambientes de Uruguay. En: A. Brazeiro (Ed.), Eco-regiones de Uruguay: Biodiversidad, presiones y conservación. Aportes a la Estrategia Nacional de Biodiversidad. (32-45). Montevideo, Uruguay: Facultad de Ciencias (FCIEN/Vida Silvestre/ SZU/CIEDUR).

Pérez-Quesada, A. \& Brazeiro, A. (2013). Contribution of rarity and commonness to patterns of species richness in biogeographic transitions regions: Woody plants of Uruguay. Austral Ecology, 38(6), 639-645.

Pessoa, E. \& Alves, M. (2019). Taxonomic Revision of Campylocentrum Sect. Laevigatum E.M. Pessoa \& M.W.Chase (Orchidaceae-Vandae-Angraecinae). Systematic Botany, 44(1), 115-132.

Pridgeon, A. M., Cribb, P. J., Chase, M. W. \& Rasmussen, F. N. (Eds.). (1999). Genera Orchidacearum vol. 1. New York: Oxford University Press.

Pridgeon, A. M., Cribb, P. J., Chase, M. W. \& Rasmussen, F. N. (2003). Genera Orchidacearum, vol. 3: Orchidoideae (part 2), Vanilloideae. Oxford, U.K: Oxford University Press.

Radins, J. A., Salazar, G. A., Cabrera, L. I., Jimenez-Machorro, R. \& Batista, J. A. N. (2014). A new paludicolous species of Malaxis (Orchidaceae) from Argentina and Uruguay. Phytotaxa, 175(3), 121-132.

Reflora. (2020). Plantas do Brasil: Resgate Histórico e Herbário Virtual para o Conhecimento e Conservação da Flora Brasileira. Recuperado de: http://reflora.jbrj.gov.br/ (Acceso Julio de 2020).

Rivas, M. (2010). Valorización y conservación de la biodiversidad en Uruguay. En: F. García-Prechac, O. Ernst, P. Arbeletche, M. Pérez-Bidegain, C. Pritsch, A. Ferenczi \& M. Rivas (Eds.), Intensificación agrícola: oportunidades y amenazas para un país productivo y natural. (89-109). Montevideo, Uruguay: Comisión Sectorial de Investigación Científica.

Rossado, A. J., Mai, P. L., Bonifacino, J. M. \& Waechter, J. L. (2014). Acianthera hygrophila (Orchidaceae), nuevo registro para Uruguay. Bonplandia, 23(2), 143-150.

Salazar, G. A., Batista, J. A. N., Cabrera, L. I., van den Berg, C., Whitten, W. M., Smidt, E. C., Buzatto, C. R., Singer, R. B., Gerlach, G., Jimenez-Machorro, R., Radins, J. A., Insaurralde, I. S., Guimaraes, L. R. S., de Barros, F., Tobar, F., Linares, J. L., Mújica, E., Dressler, R. L., Blanco, M. A., Hágsater, E. \& Chase, M. W. (2018). Phylogenetic systematics of subtribe Spiranthinae (Orchidaceae: Orchidoideae: Cranichideae) based on nuclear and plastid DNA sequences of a nearly complete generic sample. Botanical Journal of the Linnean Society, 186(3), 273-303.

Salazar, G., Cabrera, L., Madrinán, S. \& Chase, M. W. (2009). Phylogenetic relationships of Cranichidinae and Prescottiinae (Orchidaceae, Cranichideae) inferred from plastid and nuclear DNA sequences. Annals of Botany, 104(3), 403-416.

Sanguinetti, A. (2015). Habenaria parviflora (Orchidaceae), extension of its austral distributional limit and new synonymy. Nordic Journal of Botany, 33, 361-365.

Schinini, A. (2010). Orquídeas nativas del Paraguay. Asunción: Universidad Nacional de Asunción.

Schrag, A. M., Zaccagnini, M., Calamari, N. \& Canavelli, S. (2009). Climate and land-use influences on avifauna in central Argentina: Broad-Scale patterns and implications of agricultural conversion for biodiversity. Agriculture, Ecosystems and Environment, 132(1-2) 135-142.

Sistema Nacional de Áreas Protegidas (SNAP) (2020). Nodos Áreas Protegidas. Recuperado de: http://www.snap.gub.uy/ sisnap/web/mapa_conceptual/snap (Acceso Julio de 2020).

Soriano, A. (1992). Río de la Plata Grasslands. In: R. T. Coupland (Ed.), Grassland ecosystems of the world: analysis of grasslands and their uses (367-407). Cambridge, U.K: Cambridge University Press.

Swarts, N. D. \& Dixon, K. W. (2009). Terrestrial Orchid conservation in the age of extinction. Annals of Botany, 104(3), $543-556$

Szlachetko, D. L. (1996). Studies on Spirantheae [Orchidaceae]: I. Varia. Fragmenta Floristica et Geobotanica, 41(2), $845-863$.

Szlachetko, D. L., González-Tamayo, R. \& Rutkowski, P. (2001). Pachygenium, a new genus of the subtribe Cyclopogoninae (Orchidaceae). Polish Botanical Journal, 46, 3-6.

Tálamo, A., Trucco Aleman, C. E. \& Caziani, S. M. (2009). Vegetación leñosa de un camino abandonado del Chaco semiarido en relación a la matriz de vegetación circundante y el pastoreo. Ecología Austral, 19(2), 157-165.

Tan, B. C., \& Chin, C. F. (2015). Vanilla planifolia: an economically important orchid and its propagation. Minerva Biotecnologica, 27(2), 107-116.

The Plant List. (2020). Version 1.1. Recuperado de: http://www.theplantlist.org/ (Acceso Julio de 2020).

Tropicos. (2020). Missouri Botanical Garden. Recuperado de: www.tropicos.org(Acceso Julio/2020).

Zhao, W. Y., Li, J. L. \& Qi, J. G. (2007). Changes in vegetation diversity and structure in response to heavy grazing pressure in the northern Tianshan Mountains, China. Journal of Arid Environments, 68(3), 465-479. 
ANEXo 1. Especies encontradas en los diferentes tipos de ambientes presentes en el país [Bosque Ribereño (BR), Bosque de

Parque (BP), Bosque Serrano (BS), Bosque Costero (BC), Palmares (BPa) Humedales (H), Pastizales (P)].

\begin{tabular}{|c|c|}
\hline Ambientes & Especies \\
\hline BR & $\begin{array}{l}\text { Acianthera hygrophila, A. pubescens, A. sonderiana, Bipinnula penicillata, Brachystele arechavaletae, } \\
\text { B. camporum, B. dilatata, Campylocentrum densiflorum, Capanemia micromera, C. superflua, Chloraea } \\
\text { membranacea, Cyclopogon apricus, C. chloroleucus, C. elatus, C. micranthus, C. oliganthus, Galeandra } \\
\text { beyrichii, Gomesa bifolia, G. uniflora, Habenaria gourlieana, H. bractescens, H. gourlieana, H. macronectar, } \\
\text { H, paivaeana, H. parviflora, H. pentadactyla, H. repens, Pelexia arechavaletae, P. lindmanii, Skeptrostachys } \\
\text { balanophorostachya, Trichocentrum pumilum, }\end{array}$ \\
\hline$B Q$ & $\begin{array}{l}\text { Acianthera pubescens, A. sonderiana, Bipinnula gibertii, B. montana, Cyclopogon apricus, C. chloroleucus, C. } \\
\text { congestus, C. elatus, C. Iongibracteatus, Cyrtopodium brandonianum, Galeandra beyrichii, Gomesa barbata, } \\
\text { Habenaria gourlieana, H. parviflora, H. pentadactyla, Sacoila lanceolata, Skeptrostachys arechavaletanii, } \\
\text { Trichocentrum pumilum, S. gigantea }\end{array}$ \\
\hline BS & $\begin{array}{l}\text { Acianthera pubescens, A. sonderiana, Bipinnula gibertii, B. montana, B. penicillata, B. polysyka, Brachystele } \\
\text { camporum, B. cyclochila, B. dilatata, B. waldemarii, Campylocentrum densiflorum, Capanemia micromera, } \\
\text { Chloraea membranacea, Cyclopogon apricus, C. chloroleucus, C. congestus, C. elatus, C. micranthus, C. } \\
\text { taquaremboensis, Orthochilus ruwenzoriensis, Galeandra beyrichii, Gomesa bifolia, Habenaria bractescens, } \\
\text { H. gourlieana, H. macronectar, H. montevidensis, H. parviflora, H. pentadactyla, Malaxis irmae, Pelexia } \\
\text { bonariensis, Sacoila lanceolata, Skeptrostachys arechavaletanii, S. balanophorostachya, S. paraguayensis, } \\
\text { S. gigantea, S. berroana }\end{array}$ \\
\hline $\mathrm{BP}$ & $\begin{array}{l}\text { Chloraea membranacea, Cyclopogon apricus, C. congestus, C. elatus, C. oliganthus, Gomesa bifolia, } \\
\text { Habenaria bractescens, H. gourlieana, H. montevidensis, H. parviflora, H. pentadactyla, Pelexia lindmanii, } \\
\text { Skeptrostachys paraguayensis }\end{array}$ \\
\hline $\mathrm{H}$ & $\begin{array}{l}\text { Acianthera pubescens, Brachystele dilatata, Cyclopogon apricus, Habenaria exaltata, H. gourlieana, H. } \\
\text { leucosantha, H. paivaeana, H. parviflora, H. repens, Malaxis parthoni, Pelexia lindmanii, Skeptrostachys } \\
\text { paraguayensis }\end{array}$ \\
\hline $\mathrm{P}$ & $\begin{array}{l}\text { Bipinnula biplumata, B. gibertii, B. montana, B. penicillata, B. polysyka, Brachystele arechavaletae, B. } \\
\text { camporum, Chloraea membranacea, Cyclopogon apricus, Habenaria parviflora, Pelexia bonariensis, Sacoila } \\
\text { lanceolata, Skeptrostachys arechavaletanii, S. balanophorostachya, S. berroana, S. paraguayensis, S. } \\
\text { gigantea }\end{array}$ \\
\hline $\mathrm{BC}$ & $\begin{array}{l}\text { Brachystele cyclochila, B. dilatata, Chloraea membranacea, Cyclopogon apricus, C. elatus, C. micranthus, } \\
\text { Habenaria achalensis, H. gourlieana, H. paivaeana, H. parviflora, H. pentadactyla, H. platanthera, H. repens, } \\
\text { Prescottia oligantha, P. ostenii, Skeptrostachys arechavaletanii, S. berroana, S. gigantea }\end{array}$ \\
\hline Bpa & Capanemia micromera, C. superflua, Skeptrostachys balanophorostachya \\
\hline
\end{tabular}

ANEXo 2. Especies encontradas en cada departamento del país [Artigas (ART), Canelones (CAN), Cerro Largo (CER), Colonia (COL), Durazno (DUR), Flores (FLE), Florida (FLI), Lavalleja (LAV), Maldonado (MAL), Montevideo (MON), Paysandu (PAY), Rio Negro (RIO), Rivera (RIV), Rocha (ROC), Salto (SAL), San Jose (SAN), Soriano (SOR), Tacuarembo (TAC) y Treinta y Tres (TRE)].

\begin{tabular}{l|l}
\hline Departamentos & Especies \\
\hline ART & Cyclopogon apricus, C. elatus, Galeandra beyrichii, Habenaria bractescens \\
\hline CAN & $\begin{array}{l}\text { Bipinnula montana, B. penicillata, B. polysyka, Brachystele arechavaletae, B. camporum, B. dilatata, Chloraea } \\
\text { membranacea, Cyclopogon apricus, C. elatus, C. micranthus, Habenaria bractescens, H. gourlieana, H. parviflora, } \\
\text { H. pentadactyla, H. repens, Prescottia oligantha, P. ostenii, Skeptrostachys paraguayensis }\end{array}$ \\
\hline CER & $\begin{array}{l}\text { Acianthera hygrophila, A. pubescens, A. sonderiana, Bipinnula gibertii, Campylocentrum densiflorum, } \\
\text { Capanemia micromera, C. superflua, Chloraea membranacea, Cyclopogon chloroleucus, C. congestus, C. } \\
\text { elatus, C. micranthus, Gomesa bifolia, G. barbata, G. uniflora, Habenaria bractescens, H. gourlieana, H. } \\
\text { macronectar, H. montevidensis, H. paivaeana, H. parviflora Malaxis parthoni, Skeptrostachys arechavaletanii, S. } \\
\text { balanophorostachya, Trichocentrum pumilum }\end{array}$ \\
\hline COL & $\begin{array}{l}\text { Bipinnula penicillata, B. polysyka, Brachystele arechavaletae, Chloraea membranacea, C. elatus, Gomesa bifolia, } \\
\text { Habenaria bractescens, H. gourlieana, H. parviflora }\end{array}$ \\
\hline DUR & $\begin{array}{l}\text { Cyclopogon apricus, C. micranthus, Gomesa bifolia, Habenaria parviflora, Skeptrostachys balanophorostachya } \\
\text { FLE }\end{array}$ \\
\hline FLI & $\begin{array}{l}\text { Cyclopogon apricus } \\
\text { bractescens, H. gourlieana, H. parviflora }\end{array}$ \\
\hline LAV & $\begin{array}{l}\text { Bipinnula montana, B. penicillata, B. polysyka, Brachystele camporum, B. dilatata, B. waldemarii, Cyclopogon } \\
\text { apricus, C. congestus, C. elatus, C. oliganthus, C. taquaremboensis, Habenaria bractescens, H. gourlieana, H. } \\
\text { montevidensis, H. parviflora, Malaxis irmae, Skeptrostachys arechavaletanii, S. berroana, S. gigantea }\end{array}$ \\
\hline
\end{tabular}


Anexo 2. (continúa).

\begin{tabular}{|c|c|}
\hline Departamentos & Especies \\
\hline LAV & $\begin{array}{l}\text { Bipinnula montana, B. penicillata, B. polysyka, Brachystele camporum, B. dilatata, B. waldemarii, Cyclopogon } \\
\text { apricus, C. congestus, C. elatus, C. oliganthus, C. taquaremboensis, Habenaria bractescens, H. gourlieana, H. } \\
\text { montevidensis, H. parviflora, Malaxis irmae, Skeptrostachys arechavaletanii, S. berroana, S. gigantea }\end{array}$ \\
\hline MAL & $\begin{array}{l}\text { Bipinnula gibertii, B. montana, B. penicillata, B. polysica, Brachystele camporum, B. cyclochila, B. dilatata, } \\
\text { Chloraea membranacea, Cyclopogon elatus, C. apricus, C. congestus, Orthochilus ruwenzoriensis, Gomesa } \\
\text { bifolia, Habenaria bractescens, H. gourlieana, H. montevidensis, H. paivaeana, H. parviflora, H. pentadactyla, H. } \\
\text { platanthera, H. repens, Skeptrostachys arechavaletanii, S. balanophorostachya, S. berroana, S. gigantea }\end{array}$ \\
\hline MON & $\begin{array}{l}\text { Bipinnula biplumata, B. gibertii, B. penicillata, B. polysyka, Brachystele camporum, B. dilatata, B. pappulosa, } \\
\text { Chloraea membranacea, Cyclopogon apricus, Habenaria gourlieana, H. montevidensis, H. paivaeana, H. parviflora, } \\
\text { H. pentadactyla, H. repens, Pelexia arechavaletae }\end{array}$ \\
\hline PAY & $\begin{array}{l}\text { Bipinnula polysyka, Brachystele camporum, Habenaria parviflora, Skeptrostachys balanophorostachya, S. } \\
\text { paraguayensis }\end{array}$ \\
\hline RIO & $\begin{array}{l}\text { Bipinnula penicillata, B. polysyka, Brachystele camporum, B. dilatata, Chloraea bella, C. membranacea, C. elatus, } \\
\text { C. oliganthus, Orthochilus ruwenzoriensis, Gomesa bifolia, Habenaria gourlieana, H. macronectar, H. parviflora, } \\
\text { Pelexia lindmanii, Sacoila lanceolata, Skeptrostachys paraguayensis }\end{array}$ \\
\hline RIV & $\begin{array}{l}\text { Bipinnula gibertii, B. montana, Brachystele camporum, B. dilatata, Capanemia micromera, Cyclopogon apricus, } \\
\text { C. chloroleucus, C. elatus, C. longibracteatus, C. brandonianum, Galeandra beyrichii, Habenaria bractescens, H. } \\
\text { gourlieana, Habenaria leucosantha, H. montevidensis, Habenaria parviflora, H. pentadactyla, Sacoila lanceolata, } \\
\text { Skeptrostachys arechavaletanii, S. balanophorostachya }\end{array}$ \\
\hline $\mathrm{ROC}$ & $\begin{array}{l}\text { Brachystele arechavaletae, B. camporum, Brachystele dilatata, Capanemia micromera, Cyclopogon apricus, C. } \\
\text { elatus, C. micranthus, Gomesa bifolia, Habenaria gourlieana, H. parviflora, H. pentadactyla, H. repens, Pelexia } \\
\text { bonariensis, P. lindmanii, Skeptrostachys berroana, S. balanophorostachya, S. paraguayensis, Trichocentrum } \\
\text { pumilum }\end{array}$ \\
\hline SAL & $\begin{array}{l}\text { Cyclopogon congestus, C. elatus, Gomesa bifolia, Habenaria gourlieana, H. parviflora, Skeptrostachys balano- } \\
\text { phorostachya }\end{array}$ \\
\hline SAN & $\begin{array}{l}\text { Bipinnula penicillata, B. polysica, Brachystele camporum, B. dilatata, Chloraea membranacea, C. elatus, Gomesa } \\
\text { bifolia, Habenaria achalensis, H. gourlieana, H. montevidensis, H. paivaeana, H. parviflora, H. pentadactyla, H. } \\
\text { repens, Skeptrostachys arechavaletanii, S. balanophorostachya }\end{array}$ \\
\hline SOR & $\begin{array}{l}\text { Bipinnula penicillata, Bipinnula polysyka, Brachystele camporum, B. cyclochila, Chloraea membranacea, C. elatus, } \\
\text { Gomesa bifolia, Habenaria gourlieana, H. macronectar, H. parviflora }\end{array}$ \\
\hline TAC & $\begin{array}{l}\text { Bipinnula penicillata, Brachystele arechavaletae, B. dilatata, Capanemia micromera, Chloraea membranacea, } \\
\text { Cyclopogon apricus, C. chloroleucus, C. elatus, Gomesa bifolia, Habenaria bractescens, H. exaltata, H. gourlieana, } \\
\text { H. macronectar, H. parviflora, Pelexia bonariensis, Sacoila lanceolata, Skeptrostachys arechavaletanii, S. gigantea }\end{array}$ \\
\hline TRE & $\begin{array}{l}\text { Brachystele camporum, Campylocentrum densiflorum, Capanemia micromera, Chloraea membranacea, C. elatus, } \\
\text { Gomesa bifolia, Habenaria gourlieana, Skeptrostachys gigantea, Trichocentrum pumilum }\end{array}$ \\
\hline
\end{tabular}

Anexo 3. Especies encontradas en cada eco-región del país.

\begin{tabular}{l|l}
\hline Ecorregión & Especies \\
\hline Cuenca Sedimentara del Oeste & $\begin{array}{l}\text { Bipinnula penicillata, Brachystele arechavaletae, B. camporum, B. cyclochila, Chloraea } \\
\text { membranacea, Cyclopogon apricus, C. elatus, C. oliganthus,. Gomesa bifolia, Habenaria } \\
\text { gourlieana, H. macronectar, H. parviflora, Skeptrostachys paraguayensis }\end{array}$ \\
\hline Cuesta Basáltica & $\begin{array}{l}\text { Campylocentrum densiflorum, Capanemia micromera, Cyclopogon apricus, C. chloroleucus, } \\
\text { C. congestus, C. elatus, C. longibracteatus., Cyrtopodium brandonianum, Galeandra beyrichii, } \\
\text { Habenaria bractescens, H. gourlieana, H. parviflora, H. pentadactyla, Pelexia bonariensis, } \\
\text { Sacoila lanceolata, Skeptrostachys arechavaletanii , S. balanophorostachya, S. gigantea, S. } \\
\text { paraguayensis, }\end{array}$ \\
\hline Cuenca Sedimentaria Gondwanica & $\begin{array}{l}\text { Acianthera hygrophila, A. pubescens, A. Sonderiana, Bipinnula gibertii, B. montana , B. } \\
\text { penicillata, Brachystele camporum, B. dilatata, Campylocentrum densiflorum, Capanemia }\end{array}$ \\
& $\begin{array}{l}\text { micromera, C. Superflua, Chloraea membranacea, Cyclopogon apricus, C. Chloroleucus, C. } \\
\text { congestus, C. elatus, Gomesa barbata, G. bifolia, Habenaria bractescens , H. exaltata, H. }\end{array}$ \\
& $\begin{array}{l}\text { gourlieana, H. leucosantha, H. macronectar, H. montevidensis, H. parviflora, H. pentadactyla, } \\
\text { Sacoila lanceolata, Skeptrostachys arechavaletaniiS. balanophorostachya, S. gigantea }\end{array}$ \\
\hline Escudo Cristalino & $\begin{array}{l}\text { Bipinnula penicillata, B. polysyka, Brachystele arechavaletae, B. dilatata, Chloraea membranacea, } \\
\text { Cyclopogon apricus, C. congestus C. elatus, Habenaria bractescens, Habenaria gourlieana, H. } \\
\text { montevidensis, H. parviflora, H. repens, Skeptrostachys arechavaletanii }\end{array}$ \\
\hline Graben de Santa Lucía & $\begin{array}{l}\text { Bipinnula biplumata, B. gibertii, B. penicillata, B. polysyka, B. montana, Brachystele arechavaletae } \\
\text { B. camporum, B. dilatata, B. pappulosa Chloraea membranacea, Cyclopogon apricus, C. elatus, } \\
\text { C. micranthus, C. taquaremboensis Habenaria achalensis H. bractescens, H. gourlieana, H. } \\
\text { paivaeana, H. parviflora, H. pentadactyla, Pelexia arechavaletae, Prescottia oligantha, P. ostenii, } \\
\text { Skeptrostachys balanophorostachya, S. paraguayensis }\end{array}$ \\
\hline
\end{tabular}


Anexo 3. (continúa).

\begin{tabular}{l|l}
\hline Ecorregión & Especies \\
\hline Sierras del Este & $\begin{array}{l}\text { Bipinnula montana, B. penicillata, B. polysyka, Brachystele camporum, B. cyclochila, B. dilatata, } \\
\text { B. waldemarii, Cyclopogon apricus, C. congestus, C. elatus, C. micranthus, C. oliganthus } \\
\text { Gomesa bifolia, G. Barbata, Habenaria gourlieana, H. montevidensis, H. parviflora, Malaxis } \\
\text { irmae, Skeptrostachys balanophorostachya, S. Berroana, S. gigantea }\end{array}$ \\
\hline Graben de la Laguna Merin & $\begin{array}{l}\text { Bipinnula gibertii, Brachystele arechavaletae, B. camporum, B. cyclochila, B. dilatata, Capanemia } \\
\text { micromera, Cyclopogon apricus, Cyclopogon elatus, Orthochilus ruwenzoriensis, Gomesa }\end{array}$ \\
& bifolia, G. uniflora Habenaria gourlieana, H. paivaeana, H. parviflora, H. pentadactyla, H. \\
& platanthera, H. repens, Pelexia bonariensis, P. lindmanii, Skeptrostachys arechavaletanii, S. \\
& Balanophorostachya, S. Berroana, S. gigantea, S. paraguayensis, Trichocentrum pumilum \\
\hline
\end{tabular}

